\title{
The Moduli Spaces of Parabolic Connections with a Quadratic Differential and Isomonodromic Deformations
}

\author{
Arata KOMYO \\ Department of Mathematics, Graduate School of Science, Osaka University, \\ Toyonaka, Osaka 560-0043, Japan \\ E-mail:a-koumyou@cr.math.sci.osaka-u.ac.jp
}

Received January 23, 2018, in final form October 03, 2018; Published online October 13, 2018

https://doi.org/10.3842/SIGMA.2018.111

\begin{abstract}
In this paper, we study the moduli spaces of parabolic connections with a quadratic differential. We endow these moduli spaces with symplectic structures by using the fundamental 2-forms on the moduli spaces of parabolic connections (which are phase spaces of isomonodromic deformation systems). Moreover, we see that the moduli spaces of parabolic connections with a quadratic differential are equipped with structures of twisted cotangent bundles.
\end{abstract}

Key words: parabolic connection; quadratic differential; isomonodromic deformation; twisted cotangent bundle

2010 Mathematics Subject Classification: 14D20; 34M56

\section{Introduction}

Let $C$ be a smooth projective curve of genus $g$ (where $g \geq 2$ ). Narasimhan-Seshadri [20] showed that vector bundles on $C$ are stable if and only if they arise from irreducible unitary representations of the fundamental group of $C$. The moduli space of stable vector bundles on $C$ is equipped with a natural symplectic structure. Although the complex structure on this moduli space depends on the complex structure of $C$, the symplectic structure of this moduli space depends only on the underlying topological surface of $C$. This picture has been investigated by Atiyah-Bott [1] and Goldman [11]. There exist generalizations of this picture. One can consider the moduli space of pairs $(E, \nabla)$ where $(E, \nabla)$ is a rank $r$ vector bundle on $C$ with a holomorphic connection $\nabla$. This moduli space is equipped with a (holomorphic) symplectic structure. There exists an analytic isomorphism between the moduli space of pairs $(E, \nabla)$ and the moduli space of representations of the fundamental group of $C$ into $\mathrm{GL}(r, \mathbb{C})$ by taking a holomorphic connection to its monodromy representation. Considering the variation of this isomorphism when deforming the curve, we can define the isomonodromic foliation on the moduli space of triples $(C, E, \nabla)$. This foliation is transversal to the fibration $(C, E, \nabla) \mapsto C$ of complementary dimension. There exists a closed 2 -form on the moduli space of triples $(C, E, \nabla)$ such that the kernel of the closed 2-form coincides with the tangent spaces of leaves of the foliation and this 2-form induces a (holomorphic) symplectic structure on the moduli space of pairs $(E, \nabla)$ over a fixed curve $C$. This generalization has been investigated by Goldman [11], Hitchin [12], and Simpson [21, 22]. Moreover, this generalized picture was generalized to the singular setting by Iwasaki [17], Hitchin [13], Boalch [9], and Krichever [19]. Remark that, in the logarithmic case, Inaba-Iwasaki-Saito [16] and Inaba [15] have constructed the moduli scheme of triples $(C, E, \nabla)$ (satisfying some stability condition) and showed that the closed 2-form on this moduli scheme is algebraic. 
We recall the definitions of Lagrangian triples and Hamiltonian data, which are discussed in [3]. Let $p: X \rightarrow S$ be a smooth morphism of smooth varieties. A p-connection is an $\mathcal{O}_{X^{-}}$ linear morphism $\nabla_{S}: p^{*} \Theta_{S} \rightarrow \Theta_{X}$ such that $d p \circ \nabla_{S}=\operatorname{id}_{p^{*} \Theta_{S}}$. Here $\Theta_{S}$ and $\Theta_{X}$ are the tangent sheaves of $S$ and $X$, respectively. A $p$-connection $\nabla_{S}$ is integrable if the corresponding map $\Theta_{S} \rightarrow p_{*} \Theta_{X}$ commutes with brackets. Note that an integrable $p$-connection $\nabla_{S}$ defines an action of $\Theta_{S}$ on relative differential forms $\Omega_{X / S}$ by the Lie derivatives along horizontal vector

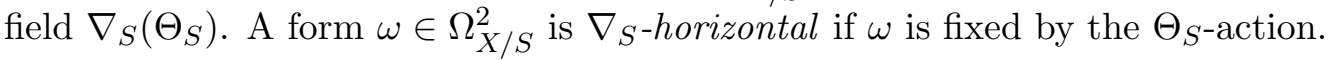

Definition 1.1. Let $X$ be a smooth algebraic variety over $\mathbb{C}$ and $T^{*}=T^{*}(X) \rightarrow X$ be the cotangent bundle on $X$. A twisted cotangent bundle on $X$ is a $T^{*}$-torsor $\pi_{\phi}: \phi \rightarrow X$ (i.e., $\pi_{\phi}$ is a fibration equipped with a simple transitive action of $T^{*}$ along the fibers) together with a symplectic form $\omega_{\phi}$ on $\phi$ such that $\pi_{\phi}$ is a polarization for $\omega_{\phi}$ (i.e., $\operatorname{dim} \phi=2 \operatorname{dim} X$ and the Poisson bracket $\{\cdot, \cdot\}$ vanishes on $\left.\pi_{\phi}^{*} \mathcal{O}_{X}\right)$ and for any 1-form $\nu$ on an open set $U \subset X$ one has $t_{\nu}^{*}\left(\omega_{\phi}\right)=\pi_{\phi}^{*} d \nu+\omega_{\phi}$ on $\pi_{\phi}^{-1}(U)$. Here $t_{\nu}: \pi_{\phi}^{-1}(U) \rightarrow \pi_{\phi}^{-1}(U) ; t_{\nu}(a)=a+\nu_{\pi(a)}$ is the translation by $\nu$.

For example, the map from the moduli space of pairs $(E, \nabla)$ to the moduli space of vector bundles defined by $(E, \nabla) \mapsto E$ is a twisted cotangent bundle on the moduli space of vector bundles (see [10, Lemma IV.4] and [7, Section 4]). Note that this moduli space of vector bundles is a smooth algebraic stack. We can define a twisted cotangent bundle on a smooth algebraic stack in the same way.

Definition 1.2. Let $S$ be a smooth variety. An $S$-Lagrangian triple consists of a morphism $\pi: X \rightarrow Y$ of $S$-varieties $p_{X}: X \rightarrow S$ and $p_{Y}: Y \rightarrow S$, a relative 2-form $\omega \in \Omega_{X / S}^{2}(X)$ and a $p_{X}$-connection $\nabla_{S}$ such that

(i) $p_{X}, p_{Y}$ and $\pi$ are smooth surjective morphisms,

(ii) the form $\omega$ is closed and non-degenerate,

(iii) for any $s \in S$ the morphism $\pi_{s}: X_{s} \rightarrow Y_{s}$ is a twisted cotangent bundle over $Y_{s}$, and

(iv) $\nabla_{S}$ is integrable and $\omega$ is $\nabla_{S}$-horizontal.

Definition 1.3. An $S$-Hamiltonian datum on an $S$-variety $p_{Y}: Y \rightarrow S$ consists of

(i) a twisted cotangent bundle $\left(\widetilde{X}, \omega_{\tilde{X}}\right), \tilde{\pi}: \widetilde{X} \rightarrow Y$ over $Y$. Put $X:=\widetilde{X} \bmod p_{Y}^{*} \Omega_{S}^{1}$ : this is a $\Theta_{Y / S}^{*}$-torsor over $Y$; let $\widetilde{X} \stackrel{r}{\rightarrow} X \stackrel{\pi}{\rightarrow} Y$ be the projections and

(ii) a section $h: X \rightarrow \widetilde{X}$ of $r$ (called Hamiltonian)

such that for each $x \in X$ the form $\left(\omega_{X}\right)_{x} \in \bigwedge^{2} \Theta_{X, x}^{*}$ has $\operatorname{rank} \operatorname{dim} X-\operatorname{dim} S$. Here we put $\omega_{X}:=h^{*} \omega_{\tilde{X}}$, which is a closed 2-form on $X$.

Remark that the twisted cotangent bundle $\tilde{X}$ over $Y$ is isomorphic to the fiber product $X \times{ }_{S} T^{*} S$ as symplectic manifolds. This isomorphism is given by the morphism $\tilde{r}: \tilde{X} \rightarrow$ $X \times{ }_{S} T^{*} S, \tilde{r}(\tilde{x})=(r(\tilde{x}), \tilde{x}-h(r(\tilde{x})))$. Here the symplectic form on $X \times{ }_{S} T^{*} S$ is equal to the sum of $\omega_{X}$ and a standard symplectic form on $T^{*} S$. Now, we describe a construction of $S$-Lagrangian triples from $S$-Hamiltonian data $\left(\widetilde{X}, \omega_{\tilde{X}}, \tilde{\pi}, h\right)$. Let $\pi: X \rightarrow Y$ be the map as in Definition 1.3. For each $s \in S$, the map $\pi_{s}: X_{s} \rightarrow Y_{s}$ is a $T^{*}\left(Y_{s}\right)$-torsor induced by the $\Theta_{Y / S^{*}}^{*}$ torsor $X \rightarrow Y$. Let $\omega$ be the image of $\omega_{X}$ under the natural morphism $\Omega_{X}^{2}(X) \rightarrow \Omega_{X / S}^{2}(X)$. For the natural map $\iota_{X_{s}}: X_{s} \rightarrow X$, the pull-back $\iota_{X_{s}}^{*} \omega$ is a symplectic form on $X_{s}$. Let $a \in \Omega_{Y_{s}}^{1}(U)$ be a local section over an open set $U \subset Y_{s}$. We take a collection $\left\{\left(U_{i}, \tilde{a}_{i}\right)\right\}_{i}$ where $\left\{U_{i}\right\}_{i}$ is an open covering of $U$ and $\left.\tilde{a}_{i} \in \Omega_{Y}^{1}\right|_{Y_{s}}\left(U_{i}\right)$ such that $\iota_{Y_{s}}^{*}\left(\tilde{a}_{i}\right)=\left.a\right|_{U_{i}}$, where $\iota_{Y_{s}}: Y_{s} \rightarrow Y$ is the natural map. We can show that $t_{a}^{*}\left(\iota_{X_{s}}^{*} h^{*} \omega_{\tilde{X}}\right)=\left(\left.h\right|_{X_{s}}\right)^{*} t_{\tilde{a}_{i}}^{*}\left(\iota_{\widetilde{X}_{s}}^{*} \omega_{\tilde{X}}\right)$ on $\pi_{s}^{-1}\left(U_{i}\right)$, where $\iota_{\tilde{X}_{s}}: \tilde{X}_{s} \rightarrow \tilde{X}$ is the natural map. (Here note that $\tilde{X}$ is isomorphic to 
$X \times{ }_{S} T^{*} S$.) In particular, the right-hand side is independent of the choice of a lift $\tilde{a}_{i}$ of $\left.a\right|_{U_{i}}$. Then $t_{a}^{*}\left(\iota_{X_{s}}^{*} \omega\right)-\iota_{X_{s}}^{*} \omega=\left(\left.h\right|_{X_{s}}\right)^{*} t_{\tilde{a}_{i}}^{*}\left(\iota_{\widetilde{X}_{s}}^{*} \omega_{\tilde{X}}\right)-\left(\left.h\right|_{X_{s}}\right)^{*}\left(\iota_{\widetilde{X}_{s}}^{*} \omega_{\tilde{X}^{\prime}}\right)=\left(\left.h\right|_{X_{s}}\right)^{*}\left(\left.\tilde{\pi}\right|_{\tilde{X}_{s}}\right)^{*} d\left(\tilde{a}_{i}\right)=\pi_{s}^{*} d a$. We have that $\pi_{s}: X_{s} \rightarrow Y_{s}$ is a twisted cotangent bundle. Put $p_{X}:=p_{Y} \circ \pi$. The kernels of $\left(\omega_{X}\right)_{x}$ for each $x \in X$ form a subbundle of the tangent bundle $T X$, which is transversal to fibers of $p_{X}$. Since the form $\left(\omega_{X}\right)_{x} \in \bigwedge^{2} \Theta_{X, x}^{*}$ has $\operatorname{rank} \operatorname{dim} X-\operatorname{dim} S$ and $\omega_{X}$ is closed, this subbundle defines an integrable $p_{X}$-connection $\nabla_{S}$. By the construction, $\omega$ is $\nabla_{S}$-horizontal. Then $\left(\pi: X \rightarrow Y, \omega, \nabla_{S}\right)$ is an $S$-Lagrangian triple. The purpose of this paper is to construct $S$ Hamiltonian data $\left(\widetilde{X}, \omega_{\tilde{X}}, \tilde{\pi}, h\right)$ from $S$-Lagrangian triples $\left(\pi: X \rightarrow Y, \omega_{X}, \nabla_{S}\right)$ by using concrete argument in the case of isomonodromic deformations. (There exists a more abstract construction in [3] for a general case.) Now, following [3], we describe that the Hamiltonian $h: X \rightarrow \widetilde{X}$ of an $S$-Hamiltonian datum is locally given by local functions and the integrable $p_{X}$-connection $\nabla_{S}$ associated to the $S$-Hamiltonian datum has a description by these functions. Let $x$ be a point of $X$. Let $y=\pi(x)$ and $s=p_{X}(x)$ be the projections of $x$. Let $\left(t_{a}\right)_{a=1, \ldots, \operatorname{dim} S}$ be local coordinates on a neighborhood of $s \in S$ and $q_{i}, i=1, \ldots, \operatorname{dim} Y_{s}$, be functions on a neighborhood of $y \in Y$ such that $\left(q_{i}, t_{a}\right)_{i, a}$ are local coordinates at $y$ on $Y$. Here we denote pull-backs of local functions by the same notations as the local functions for simplicity. Choose functions $h_{a}$ and $p_{i}$ on a neighborhood of $h(x) \in \widetilde{X}$ such that

$$
\omega_{\widetilde{X}}=\sum_{i=1}^{\operatorname{dim} Y_{s}} d p_{i} \wedge d q_{i}+\sum_{a=1}^{\operatorname{dim} S} d h_{a} \wedge d t_{a} .
$$

Then $\left(\boldsymbol{q}=\left(q_{i}\right)_{i}, \boldsymbol{p}=\left(p_{i}\right)_{i}, \boldsymbol{t}=\left(q_{a}\right)_{a}\right)$ are local coordinates at $x$ on $X$. The Hamiltonian $h: X \rightarrow \widetilde{X}$ is given by the functions $h_{a}(\boldsymbol{q}, \boldsymbol{p}, \boldsymbol{t})$. Note that

$$
\omega_{X}=\sum_{i=1}^{\operatorname{dim} Y_{s}} d p_{i} \wedge d q_{i}+\sum_{a=1}^{\operatorname{dim} S} d h_{a}(\boldsymbol{q}, \boldsymbol{p}, \boldsymbol{t}) \wedge d t_{a} .
$$

Put

$$
v_{h_{a}}=\partial_{t_{a}}+\sum_{i=1}^{\operatorname{dim} Y_{s}} \partial_{q_{i}}\left(h_{a}(\boldsymbol{q}, \boldsymbol{p}, \boldsymbol{t})\right) \partial_{p_{i}}-\partial_{p_{i}}\left(h_{a}(\boldsymbol{q}, \boldsymbol{p}, \boldsymbol{t})\right) \partial_{q_{i}} .
$$

We can check $\omega_{X}\left(\partial_{p_{i}}, v_{h_{a}}\right)=\omega_{X}\left(\partial_{q_{i}}, v_{h_{a}}\right)=0$ easily. Moreover we have $\omega_{X}\left(\partial_{t_{b}}, v_{h_{a}}\right)=0$, $a, b=1, \ldots, \operatorname{dim} S$, since for each $x \in X$ the form $\left(\omega_{X}\right)_{x} \in \bigwedge^{2} \Theta_{X, x}^{*}$ has $\operatorname{rank} \operatorname{dim} X-\operatorname{dim} S$. Then we have a description of $\nabla_{S}$ by $h_{a}(\boldsymbol{q}, \boldsymbol{p}, \boldsymbol{t}): \nabla_{S}\left(\partial_{t_{a}}\right)=v_{h_{a}}$.

In this paper, we consider an $S$-Lagrangian triples $\left(\pi: X \rightarrow Y, \omega_{X}, \nabla_{S}\right)$ associated to isomonodromic deformations of parabolic connections (which are logarithmic connection with quasiparabolic structures). The isomonodromic deformations of parabolic connections have been investigated by Inaba-Iwasaki-Saito [16] and Inaba [15]. In our case, $X$ is a moduli space of pointed smooth projective curves and parabolic connections (see [15, Theorem 2.1] and [16]), $Y$ is a moduli space of pointed smooth projective curves and quasi-parabolic bundles admitting a parabolic connection, and $S$ is a moduli space of pointed smooth projective curves. We have projections $p_{X}: X \rightarrow S, p_{Y}: Y \rightarrow S$ and $\pi: X \rightarrow Y$. The moduli space $X$ has the relative symplectic form $\omega$ over $S$ (see [15, Section 7]). The $p_{X}$-connection $\nabla_{S}$ is given by the isomonodromic deformations of parabolic connections (see [15, Proposition 8.1]). The main result of this paper is to construct the corresponding twisted cotangent bundle $\widetilde{X}$ over $Y$ by using computation of Cech cohomologies. We construct the twisted cotangent bundle with the remark in mind: The twisted cotangent bundle $\widetilde{X}$ over $Y$ is isomorphic to the fiber product $X \times{ }_{S} T^{*} S$.

Our argument is as follows. First, we consider the fiber product $X \times_{S} T^{*} S$ (which called extended phase space, see [14, Section 7]). The fiber product $X \times_{S} T^{*} S$ is the moduli space of 
(pointed smooth projective curves and) parabolic connections with a quadratic differential. We describe the tangent sheaf of $X \times_{S} T^{*} S$ and the symplectic form on $X \times_{S} T^{*} S$ by the Cech

cohomology (Propositions 3.1 and 3.6). Second, we describe the cotangent sheaf $\Omega_{Y}^{1}$ by the Cech cohomology, and we define an $\Omega_{Y}^{1}$-action on $X \times_{S} T^{*} S$ explicitly (Definition 4.3). We show that by this $\Omega_{Y}^{1}$-action and the symplectic form, $X \times_{S} T^{*} S$ is a twisted cotangent bundle over $Y$ (Theorem 4.4). The section $X \rightarrow X \times{ }_{S} T^{*} S$ given by the zero section of $T^{*} S \rightarrow S$ is the Hamiltonian of the Hamiltonian datum.

A twisted cotangent bundle over $Y$ is important for studying quantizations of isomonodromic deformations. In fact, quantizations of isomonodromic deformations may be described by using certain algebras of twisted differential operators, which are quantizations of twisted cotangent bundles (see $[3,7])$. It is expected that the results of this paper are useful to understand quantizations of isomonodromic deformations in the context of a certain algebro-geometric way such as $[15,16]$.

The organization of this paper is as follows. In Section 2, we recall basic definitions and basic facts on parabolic connections (in Section 2.1), Atiyah algebras (in Section 2.2) and twisted cotangent bundles (in Section 2.3). In Section 3, we treat moduli spaces of parabolic connections with a quadratic differential. First, we describe the tangent sheaves of these moduli spaces in terms of the hypercohomology of a certain complex. Second, we endow the moduli spaces with symplectic structures. In Section 4, we see that the moduli spaces of parabolic connections with a quadratic differential are equipped with structures of twisted cotangent bundles.

\section{Preliminaries}

\subsection{Moduli space of stable parabolic connections}

Following [15], we recall basic definitions and basic facts on parabolic connections. Let $C$ be a smooth projective curve of genus $g$. We put

$$
T_{n}:=\left\{\left(t_{1}, \ldots, t_{n}\right) \in C \times \cdots \times C \mid t_{i} \neq t_{j} \text { for } i \neq j\right\}
$$

for a positive integer $n$. For integers $e, r$ with $r>0$, we put

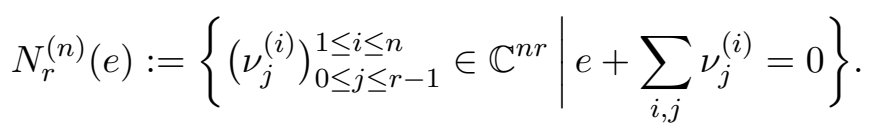

Take members $\boldsymbol{t}=\left(t_{1}, \ldots, t_{n}\right) \in T_{n}$ and $\boldsymbol{\nu}=\left(\nu_{j}^{(i)}\right)_{1 \leq i \leq n, 0 \leq j \leq r-1} \in N_{r}^{(n)}(e)$.

Definition 2.1. We say $\left(E, \nabla,\left\{l_{*}^{(i)}\right\}_{1 \leq i \leq n}\right)$ is a $(\boldsymbol{t}, \boldsymbol{\nu})$-parabolic connection of rank $r$ and degree $e$ over $C$ if

(1) $E$ is a rank $r$ algebraic vector bundle on $C$,

(2) $\nabla: E \rightarrow E \otimes \Omega_{C}^{1}\left(t_{1}+\cdots+t_{n}\right)$ is a connection, that is, $\nabla$ is a $\mathbb{C}$-linear homomorphism of sheaves satisfying $\nabla(f a)=a \otimes d f+f \nabla(a)$ for $f \in \mathcal{O}_{C}$ and $a \in E$, and

(3) for each $t_{i}, l_{*}^{(i)}$ is a filtration $\left.E\right|_{t_{i}}=l_{0}^{(i)} \supset l_{1}^{(i)} \supset \cdots \supset l_{r}^{(i)}=0 \operatorname{such}$ that $\operatorname{dim}\left(l_{j}^{(i)} / l_{j+1}^{(i)}\right)=1$ and $\left(\operatorname{res}_{t_{i}}(\nabla)-\nu_{j}^{(i)} \operatorname{id}_{\left.E\right|_{t_{i}}}\right)\left(l_{j}^{(i)}\right) \subset l_{j+1}^{(i)}$ for $j=0, \ldots, r-1$.

Remark 2.2. We have

$$
\operatorname{deg} E=\operatorname{deg}(\operatorname{det}(E))=-\sum_{i=1}^{n} \operatorname{tr}\left(\operatorname{res}_{t_{i}}(\nabla)\right)=-\sum_{i=1}^{n} \sum_{j=0}^{r-1} \nu_{j}^{(i)}=e .
$$


Definition 2.3 ([15, Definition 2.3]). Take an element $\boldsymbol{\nu} \in N_{r}^{(n)}(e)$. We call $\boldsymbol{\nu}$ special if

(1) $\nu_{j}^{(i)}-\nu_{k}^{(i)} \in \mathbb{Z}$ for some $i$ and $j \neq k$, or

(2) there exists an integer $s$ with $1<s<r$ and a subset $\left\{j_{1}^{i}, \ldots, j_{s}^{i}\right\} \subset\{0, \ldots, r-1\}$ for each $1 \leq i \leq n$ such that $\sum_{i=1}^{n} \sum_{k=1}^{s} \nu_{j_{k}^{i}}^{(i)} \in \mathbb{Z}$.

We call $\boldsymbol{\nu}$ generic if it is not special.

Take rational numbers $0<\alpha_{1}^{(i)}<\alpha_{2}^{(i)}<\cdots<\alpha_{r}^{(i)}<1$ for $i=1, \ldots, n$ satisfying $\alpha_{j}^{(i)} \neq \alpha_{j^{\prime}}^{\left(i^{\prime}\right)}$ for $(i, j) \neq\left(i^{\prime}, j^{\prime}\right)$. We choose a sufficiently generic $\boldsymbol{\alpha}=\left(\alpha_{j}^{(i)}\right)$.

Definition 2.4. A parabolic connection $\left(E, \nabla,\left\{l_{*}^{(i)}\right\}_{1 \leq i \leq n}\right)$ is $\boldsymbol{\alpha}$-stable (resp. $\boldsymbol{\alpha}$-semistable) if for any proper nonzero subbundle $F \subset E$ satisfying $\nabla(F) \subset F \otimes \Omega_{C}^{1}\left(t_{1}+\cdots+t_{n}\right)$, the inequality

$$
\frac{\operatorname{deg} F+\sum_{i=1}^{n} \sum_{j=1}^{r} \alpha_{j}^{(i)} \operatorname{dim}\left(\left(\left.F\right|_{t_{i}} \cap l_{j-1}^{(i)}\right) /\left(\left.F\right|_{t_{i}} \cap l_{j}^{(i)}\right)\right)}{\operatorname{rank} F}
$$

holds.

Let $\tilde{M}_{g, n}$ be a smooth algebraic scheme which is a certain covering of the moduli stack of $n$-pointed smooth projective curves of genus $g$ over $\mathbb{C}$ and take a universal family $\left(\mathcal{C}, \tilde{t}_{1}, \ldots, \tilde{t}_{n}\right)$ over $\tilde{M}_{g, n}$.

Definition 2.5. We denote the pull-back of $\mathcal{C}$ and $\tilde{\boldsymbol{t}}$ by the morphism $\tilde{M}_{g, n} \times N_{r}^{(n)}(e) \rightarrow \tilde{M}_{g, n}$ by the same character $\mathcal{C}$ and $\tilde{\boldsymbol{t}}=\left\{\tilde{t}_{1}, \ldots, \tilde{t}_{n}\right\}$. Then $D(\tilde{\boldsymbol{t}}):=\tilde{t}_{1}+\cdots+\tilde{t}_{n}$ becomes an effective Cartier divisor on $\mathcal{C}$ flat over $\tilde{M}_{g, n} \times N_{r}^{(n)}(e)$. We also denote by $\tilde{\boldsymbol{\nu}}$ the pull-back of the universal family on $N_{r}^{(n)}(e)$ by the morphism $\tilde{M}_{g, n} \times N_{r}^{(n)}(e) \rightarrow N_{r}^{(n)}(e)$. We define a functor $\mathcal{M}_{\mathcal{C} / \tilde{M}_{g, n}}^{\boldsymbol{\alpha}}(\tilde{\boldsymbol{t}}, r, e)$ from the category of locally noetherian schemes over $\tilde{M}_{g, n} \times N_{r}^{(n)}(e)$ to the category of sets by

$$
\mathcal{M}_{\mathcal{C} / \tilde{M}_{g, n}}^{\boldsymbol{\alpha}}(\tilde{\boldsymbol{t}}, r, e)(S):=\left\{\left(E, \nabla,\left\{l_{j}^{(i)}\right\}\right)\right\} / \sim
$$

for a locally noetherian scheme $S$ over $\tilde{M}_{g, n} \times N_{r}^{(n)}(e)$, where

(1) $E$ is a rank $r$ algebraic vector bundle on $\mathcal{C}_{S}$,

(2) $\nabla: E \rightarrow E \otimes \Omega_{\mathcal{C}_{S} / S}^{1}\left(D(\tilde{\boldsymbol{t}})_{S}\right)$ is a relative connection,

(3) for each $\left(\tilde{t}_{i}\right)_{S}, l_{*}^{(i)}$ is a filtration by subbundles $\left.E\right|_{\left(\tilde{t}_{i}\right)_{S}}=l_{0}^{(i)} \supset l_{1}^{(i)} \supset \cdots \supset l_{r}^{(i)}=0$ such that $\left(\operatorname{res}_{\left(\tilde{t}_{i}\right)_{S}}(\nabla)-\left(\tilde{\nu}_{j}^{(i)}\right)_{S} \operatorname{id}_{\left.E\right|_{t_{i}}}\right)\left(l_{j}^{(i)}\right) \subset l_{j+1}^{(i)}$ for $j=0, \ldots, r-1$, and

(4) for any geometric point $s \in S, \operatorname{dim}\left(l_{j}^{(i)} / l_{j+1}^{(i)}\right) \otimes k(s)=1$ for any $i, j$ and $\left(E, \nabla,\left\{l_{j}^{(i)}\right\}\right) \otimes k(s)$ is $\boldsymbol{\alpha}$-stable. 
Here $\left(E, \nabla,\left\{l_{j}^{(i)}\right\}\right) \sim\left(E^{\prime}, \nabla^{\prime},\left\{l_{j}^{(i)}\right\}\right)$ if there exist a line bundle $\mathcal{L}$ on $S$ and an isomorphism $\sigma: E \stackrel{\sim}{\rightarrow} E^{\prime} \otimes \mathcal{L}$ such that $\left.\sigma\right|_{t_{i}}\left(l_{J}^{(i)}\right)=l_{j}^{(i)} \otimes \mathcal{L}$ for any $i, j$ and the diagram

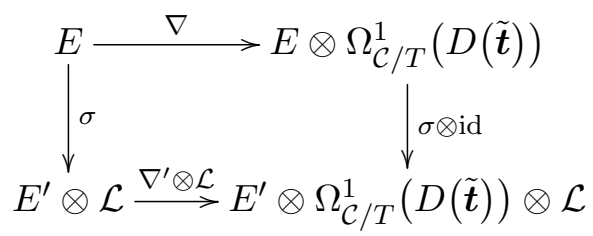

commutes.

Theorem 2.6 ([15, Theorem 2.1]). For the moduli functor $\mathcal{M}_{\mathcal{C} / \tilde{M}_{g, n}}^{\boldsymbol{\alpha}}(\tilde{\boldsymbol{t}}, r, e)$, there exists a fine moduli scheme

$$
M_{\mathcal{C} / \tilde{M}_{g, n}}^{\boldsymbol{\alpha}}(\tilde{\boldsymbol{t}}, r, e) \longrightarrow \tilde{M}_{g, n} \times N_{r}^{(n)}(e)
$$

of $\boldsymbol{\alpha}$-stable parabolic connections of rank $r$ and degree $e$, which is smooth and quasi-projective. The fiber $M_{C_{x}}^{\boldsymbol{\alpha}}\left(\tilde{\boldsymbol{t}}_{x}, \boldsymbol{\nu}\right)$ over $(x, \boldsymbol{\nu}) \in \tilde{M}_{g, n} \times N_{r}^{(n)}(e)$ is the moduli space of $\boldsymbol{\alpha}$-stable $\left(\tilde{\boldsymbol{t}}_{x}, \boldsymbol{\nu}\right)$-parabolic connections whose dimension is $2 r^{2}(g-1)+n r(r-1)+2$ if it is non-empty.

\subsection{Atiyah algebras}

Following [4, Section 1], we recall the Atiyah algebra. Let $C$ be a smooth projective curve, and $\Theta_{C}$ be the tangent sheaf. Let $E$ be a vector bundle of rank $r$ on $C$. Put $\mathcal{D}_{E}=\mathcal{D}$ iff $(E, E)=\bigcup_{i} \mathcal{D}_{i}$, $\mathcal{D}_{i}$ is the sheaf of differential operators of degree $\leq i$ on $E$. We have $\mathcal{D}_{i} / \mathcal{D}_{i-1}=\mathcal{E} \operatorname{nd}(E) \otimes S^{i}\left(\Theta_{C}\right)$ where $S^{i}\left(\Theta_{C}\right)$ is the $i$-th symmetric product of $\Theta_{C}$. Let symb ${ }_{1}: \mathcal{D}_{1} \rightarrow \mathcal{E}$ nd $(E) \otimes \Theta_{C}$ be the natural morphism $\mathcal{D}_{1} \rightarrow \mathcal{D}_{1} / \mathcal{O}_{C}=\mathcal{E} \operatorname{nd}(E) \otimes \Theta_{C}$.

Definition 2.7. We define the Atiyah algebra of $E$ as

$$
\mathcal{A}_{E}=\left\{\partial \in \mathcal{D}_{1} \mid \operatorname{symb}_{1}(\partial) \in \operatorname{id}_{E} \otimes \Theta_{C} \subset \mathcal{E} \operatorname{nd}(E) \otimes \Theta_{C}\right\} .
$$

Here, for $v \in \mathcal{D}_{1}, \operatorname{symb}_{1}(v)$ is the symbol of the differential operator $v$.

We have inclusions $\mathcal{D}_{0}=\mathcal{E} \operatorname{nd}(E) \subset \mathcal{A}_{E} \subset \mathcal{D}_{1}$ and the short exact sequence

$$
0 \longrightarrow \mathcal{E} \operatorname{nd}(E) \longrightarrow \mathcal{A}_{E} \stackrel{\text { symb }_{1}}{\longrightarrow} \Theta_{C} \longrightarrow 0 .
$$

Fix a positive integer $n$. Let $D=t_{1}+\cdots+t_{n}$ be an effective divisor of $C$ where $t_{1}, \ldots, t_{n}$ are distinct points of $C$. We put $\mathcal{A}_{E}(D):=\operatorname{symb}_{1}^{-1}\left(\Theta_{C}(-D)\right)$. Then we have the following exact sequence

$$
0 \longrightarrow \mathcal{E} \operatorname{nd}(E) \longrightarrow \mathcal{A}_{E}(D) \stackrel{\mathrm{symb}_{1}}{\longrightarrow} \Theta_{C}(-D) \longrightarrow 0 \text {. }
$$

For a connection $\nabla: E \rightarrow E \otimes \Omega_{C}^{1}(D)$, we define a splitting

$$
\iota(\nabla): \Theta_{C}(-D) \longrightarrow \mathcal{A}_{E}(D)
$$

as follows. Let $U$ be an affine open subset of $C$ where we have a trivialization $\left.E\right|_{U} \cong \mathcal{O}_{U}^{\oplus r}$. We denote by $A f^{-1} d f$ a connection matrix of $\nabla$ on $U$ where $f$ is a local defining equation of $t_{i}$ and $A \in M_{r}\left(\mathcal{O}_{U}\right)$. For an element $g \frac{\partial}{\partial f} \in \Theta_{C}(-D)(U)$, we define the element $\iota(\nabla)\left(g \frac{\partial}{\partial f}\right):=$ $g\left(\frac{\partial}{\partial f}+A f^{-1}\right) \in \mathcal{A}_{E}(D)(U)$, which gives a map $\iota(\nabla)(U): \Theta_{C}(-D)(U) \rightarrow \mathcal{A}_{E}(D)(U)$. By this map, we obtain the splitting (2.1). 


\subsection{Twisted cotangent bundles}

Following [2, Section 2], we recall the definition of $\Omega_{\bar{X}}^{\geq 1}$-torsors and recall the correspondence between twisted cotangent bundles and $\Omega_{X}^{\geq 1}$-torsors. Let $X$ be a smooth algebraic variety over $\mathbb{C}$.

Definition 2.8. Let $d: A^{n} \rightarrow A^{n+1}$ be a morphism of sheaves of abelian groups on $X$, considered as length 2 complex $A^{\bullet}$ supported in degree $n$ and $n+1$. An $A^{\bullet}$-torsor is a pair $(\mathcal{F}, c)$, where $\mathcal{F}$ is an $A^{n}$-torsor and $c: \mathcal{F} \rightarrow A^{n+1}$ is a map such that $c(a+\phi)=d(a)+c(\phi)$ for $a \in A^{n}, \phi \in \mathcal{F}$.

Let $\Omega_{X}^{\geq 1}:=\left(\Omega_{X}^{1} \rightarrow \Omega_{X}^{2 \mathrm{cl}}\right)$ be the truncated de Rham complex, where $\Omega_{X}^{2 \mathrm{cl}}$ are closed 2 -forms on $X$. For example, let $T^{*} X \rightarrow X$ be the cotangent bundle of $X$ and $\theta_{X}$ be the canonical 1-form on $T^{*} X$. The cotangent sheaf $\Omega_{X}^{1}$ of $X$ is an $\Omega_{X}^{1}$-torsor, which is trivial. For the $\Omega_{X}^{1}$-torsor $\Omega_{X}^{1}$, we define a map $c: \Omega_{X}^{1} \rightarrow \Omega_{X}^{2 c l}$ as follows. Let $U$ be a Zariski open set over $X$. We assigne $\gamma \in \Omega_{X}^{1}(U)$ (which is a section $\gamma: U \rightarrow T^{*} X$ of $T^{*} X \rightarrow X$ on $U$ ) to $\gamma^{*} d \theta_{X} \in \Omega_{X}^{2 c l}(U)$. For $\gamma, \gamma^{\prime} \in \Omega_{X}^{1}(U)$, we have $c\left(\gamma+\gamma^{\prime}\right)-c(\gamma)=\left(\gamma+\gamma^{\prime}\right)^{*} d \theta_{X}-(\gamma)^{*} d \theta_{X}=d\left(\gamma+\gamma^{\prime}\right)-d \gamma=d \gamma^{\prime}$. Then the pair $\left(\Omega_{X}^{1}, c\right)$ is an $\Omega_{X}^{\geq 1}$-torsor.

We recall the correspondence between twisted cotangent bundles and $\Omega_{\bar{X}}^{\geq 1}$-torsors. For any morphism $f: X \rightarrow Y$ between algebraic varieties, let $\Gamma(f)$ be the sheaf of set on $Y$ where $\Gamma(f)(U)$ is the set of sections of $f$ over $U$ for each open set $U \subset Y$. If $f: X \rightarrow Y$ is a $T^{*} Y$-torsor, then $\Gamma(f)$ is an $\Omega_{Y}^{1}$-torsor. We consider a twisted cotangent bundle $\pi_{\phi}: \phi \rightarrow X$. Then $\Gamma\left(\pi_{\phi}\right)$ is an $\Omega_{X}^{1}$-torsor. We define a map $c: \Gamma\left(\pi_{\phi}\right) \rightarrow \Omega_{X}^{2 \mathrm{cl}}$ by $c(\gamma):=\gamma^{*}\left(\omega_{\phi}\right)$. We have $c(a+\gamma)-c(\gamma)=$ $\gamma^{*} t_{a}^{*}\left(\omega_{\phi}\right)-\gamma^{*}\left(\omega_{\phi}\right)=d a$. Then $\left(\Gamma\left(\pi_{\phi}\right), c\right)$ is an $\Omega_{X}^{\geq 1}$-torsor. Conversely, for an $\Omega_{X}^{\geq 1}$-torsor $(\mathcal{F}, c)$, let $\pi_{\phi}: \phi \rightarrow X$ be the space of the torsor $\mathcal{F}$. The symplectic form is defined as the unique form such that for a section $\gamma \in \mathcal{F}$ of $\pi_{\phi}$ the corresponding isomorphism $T^{*} X \stackrel{\sim}{\rightarrow} \phi ; 0 \rightarrow \gamma$, identifies $\omega_{\phi}$ with $\omega+\pi^{*} c(\gamma)$. Here $\omega$ is the canonical symplectic form on the cotangent bundle $T^{*} X$.

\section{Moduli scheme of parabolic connections with a quadratic differential}

In this section, we study the moduli space of parabolic connections with a quadratic differential, which is generalization of the moduli space of parabolic connections studied by Inaba-IwasakiSaito [16] and Inaba [15]. In Section 3.2, we describe the (algebraic) tangent sheaf of this moduli space in terms of the hypercohomology of a certain complex by generalization of the description of the tangent sheaf of the moduli space of parabolic connections in $[15,16,18]$. Moreover, we describe the analytic tangent sheaf in terms of the hypercohomology of a certain analytic complex as in [15, Section 7]. This description is more simple than the algebraic one. In Section 3.3, we recall the description of the vector fields associated to the isomonodromic deformations in terms of the description of the (algebraic) tangent sheaf as in [14, Section 6] and [18, Section 3.3]. In Section 3.4, we show that the moduli space of parabolic connections with a quadratic differential is endowed with a symplectic structures. This is the main purpose of this section. In Section 3.5, we consider moduli spaces of parabolic connections with a quadratic differential as extended phase spaces of isomonodromic deformations. The classical trick of turning a time dependent Hamiltonian flow into an autonomous one by adding variables is wellknown. In this trick, the space given by adding the variables to a phase space is called an extended phase space. (Hamiltonians of isomonodromic deformations are time dependent.)

Hurtubise [14] also studied the moduli space of connections with a quadratic differential. In [14, Section 7], the moduli space of connections with a quadratic differential is decomposed locally into a product of the symplectic manifolds: the moduli space of connections on a fixed curve and the cotangent bundle of the moduli space of curves. This local decomposition is given by using the isomonodromic deformation of the "background connection", which is discussed in [14, 
Section 6]. Then we can show that the moduli space of connections with a quadratic differential is endowed with a symplectic structure locally. Moreover, in [14] the section of the map from the moduli space of connections with a quadratic differential to the moduli space of connections is defined by using the Hamiltonians defined in [14, Section 6]. On the other hand, in our argument we use the ordinary isomonodromic deformation instead of the isomonodromic deformation of the background connection. Then we have an algebraic symplectic structure on the moduli space of (parabolic) connections with a quadratic differential globally. Our corresponding section of the map from the moduli space of connections with a quadratic differential to the moduli space of connections is defined by using the zero section of the map from the moduli space of curves with a quadratic differential to the moduli space of curves.

\subsection{Moduli space of stable parabolic connections with a quadratic differential}

Let $T^{*} \tilde{M}_{g, n}$ be the total space of the cotangent bundle of $\tilde{M}_{g, n}$. We denote by $\widehat{M}_{\mathcal{C} / \tilde{M}_{g, n}}^{\alpha}(\tilde{\boldsymbol{t}}, r, e)$ the fiber product of $T^{*} \tilde{M}_{g, n} \times N_{r}^{(n)}(e)$ and $M_{\mathcal{C} / \tilde{M}_{g, n}}^{\boldsymbol{\alpha}}(\tilde{\boldsymbol{t}}, r, e)$ over $\tilde{M}_{g, n} \times N_{r}^{(n)}(e)$ :

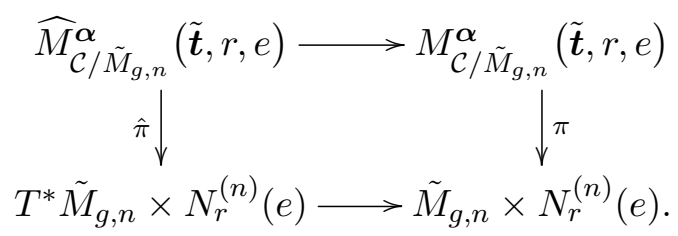

We call the fiber product $\widehat{M}_{\mathcal{C} / \tilde{M}_{g, n}}^{\boldsymbol{\alpha}}(\tilde{\boldsymbol{t}}, r, e)$ the moduli space of $\boldsymbol{\alpha}$-stable parabolic connections with a quadratic differential. If we take a zero section of $T^{*} \tilde{M}_{g, n} \rightarrow \tilde{M}_{g, n}$, then we have an inclusion

$$
M_{\mathcal{C} / \tilde{M}_{g, n}}^{\boldsymbol{\alpha}}(\tilde{\boldsymbol{t}}, r, e) \longrightarrow \widehat{M}_{\mathcal{C} / \tilde{M}_{g, n}}^{\boldsymbol{\alpha}}(\tilde{\boldsymbol{t}}, r, e)
$$

Let $(C, \boldsymbol{t}) \in \tilde{M}_{g, n}$. The tangent space of $\tilde{M}_{g, n}$ at $(C, \boldsymbol{t})$ is isomorphic to $H^{1}\left(C, \Theta_{C}(-D(\boldsymbol{t}))\right)$. By the Serre duality, the cotangent space at $(C, \boldsymbol{t})$ is isomorphic to $H^{0}\left(C, \Omega_{C}^{\otimes 2}(D(\boldsymbol{t}))\right)$, which is the space of (global) quadratic differentials on $(C, \boldsymbol{t})$.

\subsection{Infinitesimal deformations}

For simplicity, we put $\widehat{M}:=\widehat{M}_{\mathcal{C} / \tilde{M}_{g, n}}^{\alpha}(\tilde{\boldsymbol{t}}, r, e)$ and $\mathcal{C}_{\widehat{M}}:=\mathcal{C} \times_{\tilde{M}_{g, n}} \widehat{M}$. Let $\left(\tilde{E}, \tilde{\nabla},\left\{\tilde{l}_{j}^{(i)}\right\}, \tilde{\psi}\right)$ be a universal family on $\mathcal{C}_{\widehat{M}}$. Let $\mathcal{A}_{\tilde{E}}(D(\tilde{\boldsymbol{t}}))$ be the relative Atiyah algebra which is the extension

$$
0 \longrightarrow \mathcal{E} \operatorname{nd}(\tilde{E}) \longrightarrow \mathcal{A}_{\tilde{E}}(D(\tilde{\boldsymbol{t}})) \underset{\iota(\tilde{\nabla})}{\stackrel{\mathrm{symb}_{1}}{\rightleftarrows}} \Theta_{\mathcal{C}_{\widehat{M}} / \widehat{M}}(-D(\tilde{\boldsymbol{t}})) \longrightarrow 0
$$

where $\iota(\tilde{\nabla}): \Theta_{\mathcal{C}_{\widehat{M}} / \widehat{M}}(-D(\tilde{\boldsymbol{t}})) \rightarrow \mathcal{A}_{\tilde{E}}(D(\tilde{\boldsymbol{t}}))$ is the $\mathcal{O}_{X}$-linear section of symb ${ }_{1}$ associated to the relative connection $\tilde{\nabla}$. We put

$$
\begin{aligned}
& \widetilde{\mathcal{F}}^{0}:=\left\{s \in \mathcal{E} \operatorname{nd}(\tilde{E})|s|_{\tilde{t}_{i} \times \widehat{M}}\left(\tilde{l}_{j}^{(i)}\right) \subset \tilde{l}_{j}^{(i)} \text { for any } i, j\right\} \quad \text { and } \\
& \mathcal{F}^{0}:=\left\{s \in \mathcal{A}_{\tilde{E}}(D(\tilde{\boldsymbol{t}}))\left|\left(s-\iota(\tilde{\nabla}) \circ \operatorname{symb}_{1}(s)\right)\right|_{\tilde{t}_{i} \times \widehat{M}}\left(\tilde{l}_{j}^{(i)}\right) \subset \tilde{l}_{j}^{(i)} \text { for any } i, j\right\} .
\end{aligned}
$$

Then we have an extension

$$
0 \longrightarrow \widetilde{\mathcal{F}}^{0} \longrightarrow \mathcal{F}^{0} \underset{\iota(\tilde{\nabla})}{\stackrel{\mathrm{symb}_{1}}{\rightleftarrows}} \Theta_{\mathcal{C}_{\widehat{M}} / \widehat{M}}(-D(\tilde{\boldsymbol{t}})) \longrightarrow 0 .
$$


We put

$$
\begin{aligned}
& \widetilde{\mathcal{F}}^{1}:=\left\{s \in \mathcal{E} \operatorname{nd}(\tilde{E}) \otimes \Omega_{\mathcal{C}_{\widehat{M}} / \widehat{M}}^{1}(D(\tilde{\boldsymbol{t}})) \mid \operatorname{res}_{\tilde{t}_{i} \times M_{\mathcal{C} / T}^{\boldsymbol{\alpha}}(\tilde{\boldsymbol{t}}, r, e)}(s)\left(\tilde{l}_{j}^{(i)}\right) \subset \tilde{l}_{j+1}^{(i)} \text { for any } i, j\right\} \quad \text { and } \\
& \mathcal{F}^{1}:=\widetilde{\mathcal{F}}^{1} \oplus \Omega_{\mathcal{C}_{\widehat{M}} / \widehat{M}}^{\otimes 2}(D(\tilde{\boldsymbol{t}})) .
\end{aligned}
$$

We define a homomorphism $d_{\tilde{\nabla}}: \widetilde{\mathcal{F}}^{0} \rightarrow \widetilde{\mathcal{F}}^{1}$ as $s \mapsto \tilde{\nabla} \circ s-s \circ \tilde{\nabla}$ and we define a homomorphism $d_{\tilde{\psi}}: \Theta_{\mathcal{C}_{\widehat{M}} / \widehat{M}}(-D(\tilde{\boldsymbol{t}})) \rightarrow \Omega_{\mathcal{C}_{\widehat{M}} / \widehat{M}}^{\otimes 2}(D(\tilde{\boldsymbol{t}}))$ as follows. Take an affine open covering $\left\{U_{\alpha}\right\}$ of $\mathcal{C}_{\widehat{M}}$ such that we can take trivializations of $\Theta_{\mathcal{C}_{\widehat{M}} / \widehat{M}}(-D(\tilde{\boldsymbol{t}}))$ and $\Omega_{\mathcal{C}_{\widehat{M}} / \widehat{M}}(D(\tilde{\boldsymbol{t}}))$ on each $U_{\alpha}$. For an element $a \partial / \partial f_{\alpha} \in \Theta_{\mathcal{C}_{\widehat{M}} / \widehat{M}}(-D(\tilde{\boldsymbol{t}}))\left(U_{\alpha}\right)$, we define a homomorphism on $U_{\alpha}$ by

$$
a \frac{\partial}{\partial f_{\alpha}} \longmapsto\left(\frac{\partial \psi_{U_{\alpha}}}{\partial f_{\alpha}} a+2 \psi_{U_{\alpha}} \frac{\partial a}{\partial f_{\alpha}}\right) d f_{\alpha} \otimes d f_{\alpha} \in \Omega_{\mathcal{C}_{\widehat{M}} / \widehat{M}}^{\otimes 2}(D(\tilde{\boldsymbol{t}}))\left(U_{\alpha}\right)
$$

where $\left.\tilde{\psi}\right|_{U_{\alpha}}=\psi_{U_{\alpha}} d f_{\alpha} \otimes d f_{\alpha}$. By the homomorphism on each $U_{\alpha}$, we can define a homomorphism $d_{\tilde{\psi}}$. We define a complex $\mathcal{F}^{\bullet}$ by the differential $d_{\mathcal{F}} \bullet=\left(d_{\tilde{\nabla}}, d_{\tilde{\psi}}\right) \circ\left(\operatorname{Id}-\iota(\tilde{\nabla}) \circ\right.$ symb $\left._{1}, \operatorname{symb}_{1}\right)$ :

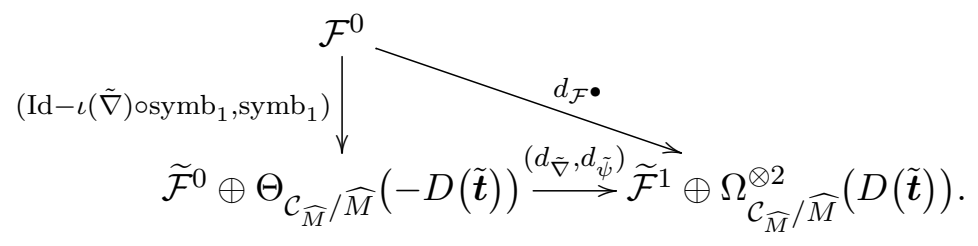

Proposition 3.1. We put $\widehat{M}=\widehat{M}_{\mathcal{C} / \tilde{M}_{g, n}}^{\boldsymbol{\alpha}}(\tilde{\boldsymbol{t}}, r, e)$ and $M=M_{\mathcal{C} / \tilde{M}_{g, n}}^{\boldsymbol{\alpha}}(\tilde{\boldsymbol{t}}, r, e)$. Let $\mathcal{F}_{M}^{0}$, $\widetilde{\mathcal{F}}_{M}^{0}$, and $\widetilde{\mathcal{F}}_{M}^{1}$ be the pull-backs of $\mathcal{F}^{0}, \widetilde{\mathcal{F}}^{0}$, and $\widetilde{\mathcal{F}}^{1}$ by the natural immersion $\mathcal{C}_{M} \rightarrow \mathcal{C}_{\widehat{M}}$, respectively. There exist canonical isomorphisms

$$
\begin{aligned}
& \hat{\varsigma}: \Theta_{\widehat{M} / N_{r}^{(n)}(e)} \stackrel{\sim}{\longrightarrow} \mathbf{R}^{1}\left(\pi_{\widehat{M}}\right)_{*}\left(\mathcal{F}^{\bullet}\right), \\
& \tilde{\varsigma}: \Theta_{M / N_{r}^{(n)}(e)} \stackrel{\sim}{\longrightarrow} \mathbf{R}^{1}\left(\pi_{M}\right)_{*}\left(\mathcal{F}_{M}^{0} \rightarrow \widetilde{\mathcal{F}}_{M}^{1}\right), \quad \text { and } \\
& \varsigma: \Theta_{M /\left(\tilde{M}_{g, n} \times N_{r}^{(n)}(e)\right)} \stackrel{\sim}{\longrightarrow} \mathbf{R}^{1}\left(\pi_{M}\right)_{*}\left(\widetilde{\mathcal{F}}_{M}^{0} \rightarrow \widetilde{\mathcal{F}}_{M}^{1}\right),
\end{aligned}
$$

where $\pi_{\widehat{M}}: \mathcal{C}_{\widehat{M}} \rightarrow \widehat{M}$ and $\pi_{M}: \mathcal{C}_{M} \rightarrow M$ are the natural morphisms.

Proof. We show the existence of the isomorphism $\hat{\varsigma}$. For the existence of the isomorphisms $\tilde{\varsigma}$ and $\varsigma$, see the proof of [18, Proposition 3.2] and the proof of [15, Theorem 2.1]. We take an affine open set $\widehat{U} \subset \widehat{M}$. Let $\left(\tilde{E}, \tilde{\nabla},\left\{\tilde{l}_{j}^{(i)}\right\}, \tilde{\psi}\right)$ be the family on $\mathcal{C} \times \tilde{M}_{g, n} \widehat{U}$. We take an affine open covering $\mathcal{C}_{\widehat{U}}=\bigcup_{\alpha} U_{\alpha}$ such that $\phi_{\alpha}:\left.\tilde{E}\right|_{U_{\alpha}} \stackrel{\sim}{\rightarrow} \mathcal{O}_{U_{\alpha}}^{\oplus r}$ for any $\alpha, \sharp\left\{i\left|\tilde{t}_{i}\right|_{\mathcal{C}_{U}} \cap U_{\alpha} \neq \varnothing\right\} \leq 1$ for any $\alpha$ and $\sharp\left\{\alpha\left|\tilde{t}_{i}\right|_{\mathcal{C}_{U}} \cap U_{\alpha} \neq \varnothing\right\} \leq 1$ for any $i$. Take a relative tangent vector field $v \in \Theta_{\widehat{M} / N_{r}^{(n)}(e)}(\widehat{U})$. The field $v$ corresponds to a member $\left(\left(C_{\epsilon}, \boldsymbol{t}_{\epsilon}, \psi_{\epsilon}\right),\left(E_{\epsilon}, \nabla_{\epsilon},\left\{\left(l_{\epsilon}\right)_{j}^{(i)}\right\}\right)\right) \in \widehat{M}\left(\operatorname{Spec} \mathcal{O}_{\widehat{U}}[\epsilon]\right)$ such that $\left(\left(C_{\epsilon}, \boldsymbol{t}_{\epsilon}, \psi_{\epsilon}\right),\left(E_{\epsilon}, \nabla_{\epsilon},\left\{\left(l_{\epsilon}\right)_{j}^{(i)}\right\}\right)\right) \otimes \mathcal{O}_{\widehat{U}}[\epsilon] /(\epsilon) \cong\left(\left(\mathcal{C}_{\widehat{U}}, \boldsymbol{t}_{\widehat{U}}, \tilde{\psi}\right),\left(\tilde{E}, \tilde{\nabla},\left\{\tilde{l}_{j}^{(i)}\right\}\right)\right)$, where $\mathcal{O}_{\widehat{U}}[\epsilon]=$ $\mathcal{O}_{\widehat{U}}[t] /\left(t^{2}\right)$. Here,

- $\psi_{\epsilon} \in H^{0}\left(C_{\epsilon}, \Omega_{C_{\epsilon} / \widehat{U}}^{\otimes 2}\left(\log \left(D(\tilde{\boldsymbol{t}})_{\mathcal{O}_{\widehat{U}}[\epsilon]}\right)\right)\right)$, and

- $\nabla_{\epsilon}: E_{\epsilon} \rightarrow E_{\epsilon} \otimes \Omega_{C_{\epsilon} / \widehat{U}}^{1}\left(\log \left(D(\tilde{\boldsymbol{t}})_{\mathcal{O}_{\widehat{U}}[\epsilon]}\right)\right)$ is a connection, 
where we define the sheaf $\Omega_{C_{\epsilon} / \widehat{U}}^{1}\left(\log \left(D(\tilde{\boldsymbol{t}})_{\mathcal{O}_{\widehat{U}}[\epsilon]}\right)\right)$ as the coherent subsheaf of $\Omega_{C_{\epsilon} / \widehat{U}}^{1}\left(D(\tilde{\boldsymbol{t}})_{\mathcal{O}_{\widehat{U}}[\epsilon]}\right)$ locally generated by $f^{-1} d f$ and $d \epsilon$ for a local defining equation $f$ of $D(\tilde{\boldsymbol{t}})_{\mathcal{O}_{\widehat{U}}[\epsilon]}$ which is the pull-back of $D(\tilde{\boldsymbol{t}})$ by the morphism $C_{\epsilon} \rightarrow \mathcal{C}_{\widehat{U}} \rightarrow \mathcal{C}$. Set $U_{\alpha}^{\epsilon}:=U_{\alpha} \times \operatorname{Spec} \mathcal{O}_{\widehat{U}}[\epsilon]$. Let

$$
\mu_{\alpha \beta}(\epsilon): U_{\alpha \beta} \times \operatorname{Spec} \mathcal{O}_{\widehat{U}}[\epsilon] \stackrel{\sim}{\longrightarrow} U_{\alpha \beta} \times \operatorname{Spec} \mathcal{O}_{\widehat{U}}[\epsilon]
$$

be an isomorphism associated to the first-order deformation $C_{\epsilon}$ of $\mathcal{C}_{\widehat{U}}$. The isomorphism $\mu_{\alpha \beta}(\epsilon)$ satisfies

$$
\mu_{\alpha \beta}(\epsilon)^{*}(\epsilon)=\epsilon, \quad \mu_{\alpha \beta}(\epsilon)^{*}(f)=f+\epsilon d_{\alpha \beta} f, \quad \text { for } \quad f \in \mathcal{O}_{U_{\alpha \beta}},
$$

for some $d_{\alpha \beta} \in \Theta_{\mathcal{C}_{\widehat{U}}}(-D)\left(U_{\alpha \beta}\right)$. We describe $d_{\alpha \beta}$ as $d_{\alpha \beta}=\frac{\partial \mu_{\alpha \beta}(\epsilon)}{\partial \epsilon} \frac{\partial}{\partial f_{\alpha}} \in \Theta_{\mathcal{C}_{\widehat{U}}}(-D)\left(U_{\alpha \beta}\right)$. Here, $f_{\alpha}$ is a local defining equation of $\left.\tilde{t}_{i}\right|_{\widehat{U}} \cap U_{\alpha}$. Set $\phi_{\alpha}^{\epsilon}:\left.E_{\epsilon}\right|_{U_{\alpha}^{\epsilon}} \cong \mathcal{O}_{U_{\alpha}^{\epsilon}}^{\oplus r}$. There is an isomorphism

$$
\varphi_{\alpha}:\left.\left.\quad E_{\epsilon}\right|_{U_{\alpha}^{\epsilon}} \stackrel{\phi_{\alpha}^{\epsilon}}{\sim} \mathcal{O}_{U_{\alpha}^{\epsilon}}^{\oplus r} \stackrel{\phi_{\alpha}^{-1}}{\sim} \tilde{E}\right|_{U_{\alpha}} \otimes \mathcal{O}_{\widehat{U}}[\epsilon]
$$

such that $\varphi_{\alpha} \otimes \mathcal{O}_{\widehat{U}}[\epsilon] /(\epsilon): E_{\epsilon} \otimes \mathcal{O}_{\widehat{U}}[\epsilon] /\left.\left.(\epsilon)\right|_{U_{\alpha}} \stackrel{\sim}{\rightarrow} \tilde{E}\right|_{U_{\alpha}} \otimes \mathcal{O}_{\widehat{U}}[\epsilon] /(\epsilon)=\left.\tilde{E}\right|_{U_{\alpha}}$ is the given isomorphism and that $\left.\varphi_{\alpha}\right|_{t_{i} \otimes \mathcal{O}_{\widehat{U}}[\epsilon]}\left(\left(l_{\epsilon}\right)_{j}^{(i)}\right)=\left.\tilde{l}_{j}^{(i)}\right|_{U_{\alpha} \times \operatorname{Spec} \mathcal{O}_{\widehat{U}}[\epsilon]}$ if $\left.\tilde{t}_{i}\right|_{\mathcal{C}_{\widehat{U}}} \cap U_{\alpha} \neq \varnothing$. Put

$$
\theta_{\alpha \beta}(\epsilon):\left.\mathcal{O}_{U_{\alpha \beta}^{\epsilon}}^{\oplus r} \stackrel{\left.\left(\phi_{\beta}^{\epsilon}\right)^{-1}\right|_{U_{\alpha \beta}^{\epsilon}}}{\sim} E_{\epsilon}\right|_{U_{\alpha \beta}^{\epsilon}} \stackrel{\left.\phi_{\alpha}^{\epsilon}\right|_{U_{\alpha \beta}^{\epsilon}}}{\sim} \mathcal{O}_{U_{\alpha \beta}^{\epsilon}}^{\oplus r}
$$

which is an element of $\mathcal{E} \operatorname{nd}\left(\mathcal{O}_{U_{\alpha \beta}^{\epsilon}}^{\oplus r}\right)\left(U_{\alpha \beta}^{\epsilon}\right)$. We denote $\theta_{\alpha \beta}(\epsilon)$ by

$$
\theta_{\alpha \beta}(\epsilon)=\tilde{\theta}_{\alpha \beta}+\epsilon \frac{\partial \theta_{\alpha \beta}(\epsilon)}{\partial \epsilon}, \quad \text { where } \quad \tilde{\theta}_{\alpha \beta}, \frac{\partial \theta_{\alpha \beta}(\epsilon)}{\partial \epsilon} \in \mathcal{E} \operatorname{nd}\left(\mathcal{O}_{U_{\alpha \beta} r}^{\oplus r}\right)\left(U_{\alpha \beta}\right)
$$

Set

$$
\eta_{\alpha \beta}:=\frac{\partial \theta_{\alpha \beta}(\epsilon)}{\partial \epsilon}\left(\tilde{\theta}_{\alpha \beta}\right)^{-1} \in \mathcal{E} \operatorname{nd}\left(\mathcal{O}_{U_{\alpha \beta} r}^{\oplus r}\right)\left(U_{\alpha \beta}\right)
$$

We define elements $u_{\alpha \beta} \in \mathcal{F}^{0}\left(U_{\alpha \beta}\right)$ and $\left(v_{\alpha}, w_{\alpha}\right) \in \mathcal{F}^{1}\left(U_{\alpha}\right)$ by

$$
\begin{aligned}
& u_{\alpha \beta}:=\left.\left(\left.\phi_{\alpha}\right|_{U_{\alpha \beta}}\right)^{-1} \circ \epsilon\left(d_{\alpha \beta}+\eta_{\alpha \beta}\right) \circ \phi_{\alpha}\right|_{U_{\alpha \beta}}, \\
& v_{\alpha}:=\left.\left(\varphi_{\alpha} \otimes \mathrm{id}\right) \circ \nabla_{\epsilon}\right|_{U_{\alpha}^{\epsilon}} \circ \varphi_{\alpha}^{-1}-\left.\tilde{\nabla}\right|_{U_{\alpha}^{\epsilon}} \bmod d \epsilon, \\
& w_{\alpha}:=\left.\psi_{\epsilon}\right|_{U_{\alpha}^{\epsilon}}-\left.\tilde{\psi}\right|_{U_{\alpha}^{\epsilon}} \bmod d \epsilon,
\end{aligned}
$$

respectively. We can see that

$$
u_{\beta \gamma}-u_{\alpha \gamma}+u_{\alpha \beta}=0, \quad \text { and } \quad d_{\mathcal{F}} \cdot\left(u_{\alpha \beta}\right)=\left(v_{\beta}, w_{\beta}\right)-\left(v_{\alpha}, w_{\alpha}\right) .
$$

Then $\left[\left\{u_{\alpha \beta}\right\},\left\{\left(v_{\alpha}, w_{\alpha}\right)\right\}\right]$ determines an element $\sigma_{\widehat{U}}(v)$ of $\mathbf{H}^{1}\left(\mathcal{F}_{\widehat{U}}^{\bullet}\right)$. We can check that $v \mapsto \sigma_{\widehat{U}}(v)$ determines an isomorphism

$$
\Theta_{\widehat{M} / N_{r}^{(n)}(e)}(\widehat{U}) \stackrel{\sim}{\longrightarrow} \mathbf{H}^{1}\left(\mathcal{F}_{\widehat{U}}^{\bullet}\right), \quad v \longmapsto \sigma_{\widehat{U}}(v)
$$

We denote by $\hat{\varsigma}_{\widehat{U}}$ this isomorphism. The isomorphism $\hat{\varsigma}_{\widehat{U}}$ induces the desired isomorphism $\hat{\varsigma}$. 
We describe the analytic tangent sheaf in terms of the hypercohomology of a certain analytic complex. Let $\boldsymbol{\nu}$ be an element of $N_{r}^{(n)}(e)$. Put $\widehat{M}_{\boldsymbol{\nu}}=\widehat{M}_{\mathcal{C} / \tilde{M}_{g, n}}^{\boldsymbol{\alpha}}(\tilde{\boldsymbol{t}}, r, e)_{\boldsymbol{\nu}}$, which is the fiber of $\boldsymbol{\nu}$ under $\widehat{M}_{\mathcal{C} / \tilde{M}_{q, n}}^{\boldsymbol{\alpha}}(\tilde{\boldsymbol{t}}, r, e) \rightarrow N_{r}^{(n)}(e)$. Let $j: \mathcal{C}_{\widehat{M}_{\nu}} \backslash\left\{\tilde{t}_{1}, \ldots, \tilde{t}_{n}\right\}_{\widehat{M}_{\boldsymbol{\nu}}} \rightarrow \mathcal{C}_{\widehat{M}_{\nu}}$ be the canonical inclusion. Let $\widehat{\boldsymbol{V}}:=\left.\operatorname{Ker} \tilde{\nabla}^{\mathrm{an}}\right|_{\mathcal{C}_{\widehat{M}_{\boldsymbol{\nu}}} \backslash\left\{\tilde{t}_{1}, \ldots, \tilde{t}_{n}\right\}_{\widehat{M}_{\boldsymbol{\nu}}}}$ be the locally constant sheaf of the locally free $\left(\pi_{\widehat{M}_{\nu}} \circ j\right)^{-1} \mathcal{O}_{\widehat{M}_{\nu}}$-module associated to the relative analytic connection $\tilde{\nabla}^{\text {an }}$ on $\mathcal{C}_{\widehat{M}_{\nu}} \backslash\left\{\tilde{t}_{1}, \ldots, \tilde{t}_{n}\right\}_{\widehat{M}_{\nu}}$, where $\pi_{\widehat{M}_{\nu}}: \mathcal{C}_{\widehat{M}_{\nu}} \rightarrow \widehat{M}_{\nu}$ is the natural map.

Assume that $\boldsymbol{\nu}$ is generic. We define a complex $\left(\widehat{\mathcal{F}}^{\bullet}\right)^{\text {an }}$ by

$$
\left(\widehat{\mathcal{F}}^{\bullet}\right)^{\text {an }}: j_{*}(\mathcal{E} \operatorname{nd}(\widehat{\boldsymbol{V}})) \oplus \Theta_{\mathcal{C}_{\widehat{M}_{\boldsymbol{\nu}}} / \widehat{M}_{\boldsymbol{\nu}}}(-D(\tilde{\boldsymbol{t}})) \stackrel{d_{\tilde{\psi}^{\circ}} \mathrm{pr}_{2}}{\longrightarrow} \Omega_{\mathcal{C}_{\widehat{M}_{\boldsymbol{\nu}}}^{\otimes 2} / \widehat{M}_{\boldsymbol{\nu}}}(D(\tilde{\boldsymbol{t}})),
$$

where $\mathrm{pr}_{2}$ is the second projection. We have the following commutative diagram

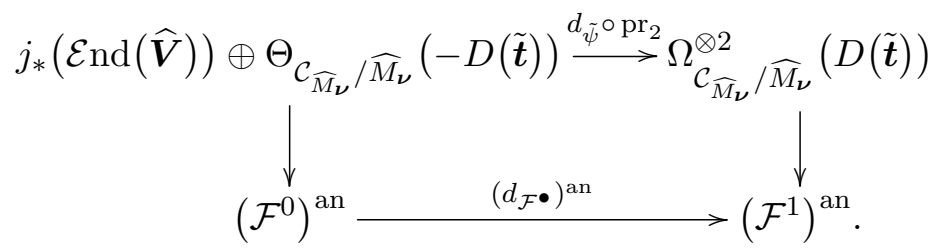

We can show that the homomorphism $\left.\operatorname{Ker} d_{\tilde{\nabla} \text { an }}\right|_{\mathcal{C}_{\widehat{M}_{\nu}}} \rightarrow j_{*}(\mathcal{E}$ nd $(\widehat{\boldsymbol{V}}))$ is an isomorphism and the homomorphism $d_{\tilde{\nabla}_{\text {an }}}:\left(\widetilde{\mathcal{F}}^{0}\right)^{\text {an }} \rightarrow\left(\widetilde{\mathcal{F}}^{1}\right)^{\text {an }}$ is surjective as in the proof of [15, Proposition 7.3]. Then we have the following proposition.

Proposition 3.2. If $\boldsymbol{\nu}$ is generic, then we have

$$
\mathbf{R}^{1}\left(\pi_{\widehat{M}_{\nu}}\right)_{*}\left(\left(\mathcal{F}^{\bullet}\right)^{\text {an }}\right) \stackrel{\sim}{\longrightarrow} \mathbf{R}^{1}\left(\pi_{\widehat{M}_{\nu}}\right)_{*}\left(\left(\widehat{\mathcal{F}}^{\bullet}\right)^{\text {an }}\right),
$$

where $\pi_{\widehat{M}_{\nu}}: \mathcal{C}_{\widehat{M}_{\nu}} \rightarrow \widehat{M}_{\nu}$ is the natural map.

\subsection{Isomonodromic deformations}

Let $\boldsymbol{\nu}$ be an element of $N_{r}^{(n)}(e)$. Put $M_{\boldsymbol{\nu}}=M_{\mathcal{C} / \tilde{M}_{g, n}}^{\boldsymbol{\alpha}}(\tilde{\boldsymbol{t}}, r, e)_{\boldsymbol{\nu}}$ which is the fiber of $\boldsymbol{\nu}$ under $M_{\mathcal{C} / \tilde{M}_{g, n}}^{\boldsymbol{\alpha}}(\tilde{\boldsymbol{t}}, r, e) \rightarrow N_{r}^{(n)}(e)$. Let $j: \mathcal{C}_{M_{\nu}} \backslash\left\{\tilde{t}_{1}, \ldots, \tilde{t}_{n}\right\}_{M_{\nu}} \rightarrow \mathcal{C}_{M_{\nu}}$ be the canonical inclusion. Let $\left.\operatorname{Ker} \tilde{\nabla}^{\mathrm{an}}\right|_{\mathcal{C}_{M_{\nu}} \backslash\left\{\tilde{t}_{1}, \ldots, \tilde{t}_{n}\right\}_{M_{\nu}}}$ be the locally constant sheaf of the locally free $\left(\pi_{M_{\nu}} \circ j\right)^{-1} \mathcal{O}_{M_{\nu^{-}}}$ module associated to the relative analytic connection $\tilde{\nabla}^{\text {an }}$ on $\mathcal{C}_{M_{\nu}} \backslash\left\{\tilde{t}_{1}, \ldots, \tilde{t}_{n}\right\}_{M_{\nu}}$, where $\pi_{M_{\nu}}: \mathcal{C}_{M_{\nu}} \rightarrow M_{\nu}$ is the natural map.

Definition 3.3. For $\pi_{\boldsymbol{\nu}}: M_{\boldsymbol{\nu}} \rightarrow \tilde{M}_{g, n}$, we say a complex foliation $\mathcal{F}$ is a foliation determined by the isomonodromic deformations if

(1) $\mathcal{F}$ is transverse to each fiber $\left(M_{\boldsymbol{\nu}}\right)_{t}=\pi_{\nu}^{-1}(t), t \in \tilde{M}_{g, n}$, and

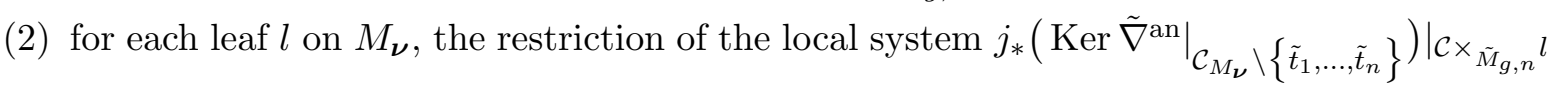
is constant.

Let $\mu: \pi_{\nu}^{*} \Theta_{\tilde{M}_{g, n}} \rightarrow R^{1}\left(\pi_{M_{\nu}}\right)_{*}\left(\Theta_{\mathcal{C}_{M_{\nu} / M_{\nu}}}(-D(\tilde{\boldsymbol{t}}))\right)$ be the Kodaira-Spencer map, where $\pi_{M_{\nu}}$ : $\mathcal{C}_{M_{\nu}} \rightarrow M_{\nu}$ is the natural morphism. We define a splitting $\mathfrak{D}$ of the tangent map $\Theta_{M_{\nu}} \rightarrow$ $\pi_{\nu}^{*}\left(\Theta_{\tilde{M}_{g, n}}\right)$ as follows

$$
\mathfrak{D}: \pi_{\nu}^{*}\left(\Theta_{\tilde{M}_{g, n}}\right) \longrightarrow \Theta_{M_{\nu}} \cong \mathbf{R}^{1}\left(\pi_{M_{\nu}}\right)_{*}\left(\mathcal{F}_{M_{\nu}}^{0} \rightarrow \widetilde{\mathcal{F}}_{M_{\nu}}^{1}\right), \quad v \longmapsto\left[\left\{\iota(\tilde{\nabla})\left(d_{\alpha \beta}\right)\right\},\{0\}\right],
$$

where $\left[\left\{d_{\alpha \beta}\right\}\right]$ is a description of $\mu(v)$ by the Čech cohomology. Here, we take an affine open covering $\left\{U_{\alpha}\right\}$. 
Proposition 3.4 ([14, Section 6], [15, Section 8], [18, Section 3.3]). The subsheaf $\mathfrak{D}\left(\pi^{*}\left(\Theta_{\tilde{M}_{g, n}}\right)\right)$ determines the foliation determined by the isomonodromic deformations.

We can take a natural lift $\widehat{\mathfrak{D}}: \hat{\pi}_{\nu}^{*}\left(\Theta_{T^{*} \tilde{M}_{g, n}}\right) \rightarrow \Theta_{\widehat{M}_{\nu}}$ of $\mathfrak{D}: \pi_{\nu}^{*}\left(\Theta_{\tilde{M}_{g, n}}\right) \rightarrow \Theta_{M_{\nu}}$ as follows. We define a complex $\mathcal{G}^{\bullet}$ by

$$
\Theta_{\mathcal{C}_{\widehat{M}_{\boldsymbol{\nu}}} / \widehat{M}_{\boldsymbol{\nu}}}(-D(\tilde{\boldsymbol{t}}))=: \mathcal{G}^{0} \stackrel{d_{\tilde{\psi}}}{\longrightarrow} \mathcal{G}^{1}:=\Omega_{\mathcal{C}_{\widehat{M}_{\boldsymbol{\nu}}}^{\otimes 2} / \widehat{M}_{\boldsymbol{\nu}}}(D(\tilde{\boldsymbol{t}}))
$$

where $d_{\tilde{\psi}}$ is defined by (3.1). Then we can show that $\hat{\pi}_{\nu}^{*} \Theta_{T^{*} \tilde{M}_{g, n}} \cong \mathbf{R}^{1}\left(\pi_{\widehat{M}_{\nu}}\right)_{*}\left(\mathcal{G}^{\bullet}\right)$. We define a lift $\widehat{\mathfrak{D}}: \hat{\pi}_{\nu}^{*} \Theta_{T^{*} \tilde{M}_{g, n}} \rightarrow \Theta_{\widehat{M}_{\nu}}$ of $\mathfrak{D}$ by the following homomorphism

$$
\widehat{\mathfrak{D}}: \mathbf{H}^{1}\left(\mathcal{G}_{\widehat{U}}^{\bullet}\right) \longrightarrow \mathbf{H}^{1}\left(\mathcal{F}_{\widehat{U}}^{\bullet}\right), \quad\left[\left\{d_{\alpha \beta}\right\},\left\{w_{\alpha}\right\}\right] \longmapsto\left[\left\{\iota(\tilde{\nabla})\left(d_{\alpha \beta}\right)\right\},\left\{\left(0, w_{\alpha}\right)\right\}\right] .
$$

\subsection{Symplectic structure}

First, we recall the canonical symplectic structure $\omega_{\tilde{M}_{g, n}}$ on $T^{*} \tilde{M}_{g, n}$. Let $U$ be an affine open set of $T^{*} \tilde{M}_{g, n}$ and let $\left(\mathcal{C}_{U}, \tilde{\psi}\right)$ be a family of curves and quadratic differentials on $U$. Let $\psi_{\alpha} d f_{\alpha}^{\otimes 2}$ be the restriction of $\tilde{\psi}$ on an affine open set $U_{\alpha} \subset \mathcal{C}_{U}$. Let $\mu_{\alpha \beta}$ be the isomorphism (3.2): $f_{\alpha}=\mu_{\alpha \beta}\left(f_{\beta}\right)$. We define a 1 -form $\theta_{\tilde{M}_{g, n}}$ on $T^{*} \tilde{M}_{g, n}$ by

$$
\theta_{\tilde{M}_{g, n}}: \mathbf{H}^{1}\left(\mathcal{G}_{U}^{\bullet}\right) \longrightarrow H^{1}\left(\Omega_{\mathcal{C}_{U} / U}^{1}\right), \quad\left[\left\{d_{\alpha \beta}\right\},\left\{w_{\alpha}\right\}\right] \longmapsto\left[\left\{d_{\beta \alpha} \psi_{\alpha} \frac{\partial \mu_{\alpha \beta}}{\partial f_{\beta}} d f_{\alpha}\right\}\right]
$$

where $\mathcal{G}_{U}$ is the complex $d_{\tilde{\psi}}: \Theta_{\mathcal{C}_{U} / U}(-D(\tilde{\boldsymbol{t}})) \rightarrow \Omega_{\mathcal{C}_{U} / U}^{\otimes 2}(D(\tilde{\boldsymbol{t}}))$. The 1 -form $\theta_{\tilde{M}_{g, n}}$ is the canonical 1-form on the cotangent bundle $T^{*} \tilde{M}_{g, n}$. Let $d \theta_{\tilde{M}_{g, n}}$ be the exterior differential of $\theta_{\tilde{M}_{g, n}}$. The 2 -form $d \theta_{\tilde{M}_{g, n}}$ gives the symplectic form on the cotangent bundle $T^{*} \tilde{M}_{g, n}$.

Proposition 3.5. Let $v=\left[\left(\left\{d_{\alpha \beta}\right\},\left\{w_{\alpha}\right\}\right)\right]$ and $v^{\prime}=\left[\left(\left\{d_{\alpha \beta}^{\prime}\right\},\left\{w_{\alpha}^{\prime}\right\}\right)\right]$ be elements of $\mathbf{H}^{1}\left(\mathcal{G}_{U}^{\bullet}\right)$. The pairing

$$
\begin{aligned}
& \mathbf{H}^{1}\left(\mathcal{G}_{U}^{\bullet}\right) \otimes \mathbf{H}^{1}\left(\mathcal{G}_{U}^{\bullet}\right) \longrightarrow \mathbf{H}^{2}\left(\Omega_{\mathcal{C}_{U} / U}^{\bullet}\right), \\
& v \otimes w \longmapsto\left[\left\{2 \cdot d_{\beta \alpha} \circ d_{\beta \gamma}^{\prime} \circ \psi_{\beta}\right\},\left\{-d_{\beta \alpha} \circ w_{\beta}^{\prime}-w_{\alpha} \circ d_{\alpha \beta}^{\prime}\right\}\right]
\end{aligned}
$$

coincides with the symplectic form $d \theta_{\tilde{M}_{g, n}}$.

Proof. Let $D_{v}: \mathcal{O}_{U_{\alpha \beta}} \rightarrow \mathcal{O}_{U_{\alpha \beta}}$ be a derivation corresponding to $v$. We compute the 2-form $d \theta_{\tilde{M}_{g, n}}\left(v, v^{\prime}\right)$ as follows

$$
\begin{aligned}
D_{v} \theta_{\tilde{M}_{g, n}}\left(v^{\prime}\right)-D_{v^{\prime}} \theta_{\tilde{M}_{g, n}}(v)+\theta_{\tilde{M}_{g, n}}\left(\left[v, v^{\prime}\right]\right) \\
=D_{v^{\prime}}\left(\mu_{\beta \alpha}\right) D_{v}\left(\psi_{\alpha} \frac{\partial \mu_{\alpha \beta}}{\partial f_{\beta}}\right) d f_{\alpha}-D_{v}\left(\mu_{\beta \alpha}\right) D_{v^{\prime}}\left(\psi_{\alpha} \frac{\partial \mu_{\alpha \beta}}{\partial f_{\beta}}\right) d f_{\alpha} \\
=D_{v^{\prime}}\left(\mu_{\beta \alpha}\right) \psi_{\alpha} \frac{\partial D_{v}\left(\mu_{\alpha \beta}\right)}{\partial f_{\beta}} d f_{\alpha}-D_{v}\left(\mu_{\beta \alpha}\right) \psi_{\alpha} \frac{\partial D_{v^{\prime}}\left(\mu_{\alpha \beta}\right)}{\partial f_{\beta}} d f_{\alpha} \\
\quad+D_{v^{\prime}}\left(\mu_{\beta \alpha}\right) \frac{\partial \mu_{\alpha \beta}}{\partial f_{\beta}} D_{v}\left(\psi_{\alpha} d f_{\alpha}\right)-D_{v}\left(\mu_{\beta \alpha}\right) \frac{\partial \mu_{\alpha \beta}}{\partial f_{\beta}} D_{v^{\prime}}\left(\psi_{\alpha} d f_{\alpha}\right) \\
=-d_{\alpha \beta}^{\prime} \psi_{\alpha} \frac{\partial d_{\alpha \beta}}{\partial f_{\beta}} d f_{\beta}+d_{\alpha \beta} \psi_{\alpha} \frac{\partial d_{\alpha \beta}^{\prime}}{\partial f_{\beta}} d f_{\beta}-d_{\alpha \beta}^{\prime} w_{\alpha} \frac{\partial \mu_{\alpha \beta}}{\partial f_{\beta}} d f_{\beta}+d_{\alpha \beta} w_{\alpha}^{\prime} \frac{\partial \mu_{\alpha \beta}}{\partial f_{\beta}} d f_{\beta} .
\end{aligned}
$$


We add the exterior differential of $d_{\alpha \beta}^{\prime} d_{\alpha \beta} \psi_{\alpha}$ to the formula above

$$
\begin{gathered}
-d_{\alpha \beta}^{\prime} \psi_{\alpha} \frac{\partial d_{\alpha \beta}}{\partial f_{\beta}} d f_{\beta}+d_{\alpha \beta} \psi_{\alpha} \frac{\partial d_{\alpha \beta}^{\prime}}{\partial f_{\beta}} d f_{\beta}-d_{\alpha \beta}^{\prime} w_{\alpha} \frac{\partial \mu_{\alpha \beta}}{\partial f_{\beta}} d f_{\beta}+d_{\alpha \beta} w_{\alpha} \frac{\partial \mu_{\alpha \beta}}{\partial f_{\beta}} d f_{\beta}+d\left(d_{\alpha \beta}^{\prime} d_{\alpha \beta} \psi_{\alpha}\right) \\
=d_{\alpha \beta}\left(d_{\alpha \beta}^{\prime} d \psi_{\alpha}+2 \psi_{\alpha} \frac{\partial d_{\alpha \beta}^{\prime}}{\partial f_{\beta}} d f_{\beta}\right)-d_{\alpha \beta}^{\prime} w_{\alpha} d f_{\alpha}+d_{\alpha \beta} w_{\alpha}^{\prime} d f_{\alpha} \\
=-d_{\beta \alpha} w_{\beta}^{\prime} d f_{\beta}-d_{\alpha \beta}^{\prime} w_{\alpha} d f_{\alpha} .
\end{gathered}
$$

By the isomorphism $H^{1}\left(\Omega_{\mathcal{C}_{U} / U}^{1}\right) \cong \mathbf{H}^{2}\left(\Omega_{\mathcal{C}_{U} / U}^{\bullet}\right)$, we have this proposition.

Proposition 3.6. Take a point $\boldsymbol{\nu} \in N_{r}^{(n)}(e)$. Let $\widehat{M}_{\mathcal{C} / \tilde{M}_{g, n}}^{\boldsymbol{\alpha}}(\tilde{\boldsymbol{t}}, r, e)_{\boldsymbol{\nu}}$ be the fiber of $\boldsymbol{\nu}$ under the composition $\widehat{M}_{\mathcal{C} / \tilde{M}_{g, n}}^{\boldsymbol{\alpha}}(\tilde{\boldsymbol{t}}, r, e)_{\boldsymbol{\nu}} \rightarrow N_{r}^{(n)}(e)$. Then the fiber $\widehat{M}_{\mathcal{C} / \tilde{M}_{g, n}}^{\boldsymbol{\alpha}}(\tilde{\boldsymbol{t}}, r, e)_{\boldsymbol{\nu}}$ has an algebraic symplectic structure.

We can obtain the above proposition by the following two propositions.

Proposition 3.7. There is a non-degenerate relative 2 -form $\omega \in H^{0}\left(\widehat{M}, \Omega_{\widehat{M} / N_{r}^{(n)}(e)}^{2}\right)$.

Proof. We set $\eta(s):=s-\iota(\tilde{\nabla}) \circ \operatorname{symb}_{1}(s) \in \mathcal{E}$ nd $(\tilde{E})$, where $s \in \mathcal{F}^{0}$. For

$$
\begin{aligned}
& v=\left[\left(\left\{u_{\alpha, \beta}\right\},\left\{\left(v_{\alpha}, w_{\alpha}\right)\right\}\right)\right] \in \mathbf{H}^{1}\left(\mathcal{C} \times_{T} \widehat{U}, \mathcal{F}_{\widehat{U}}^{\bullet}\right) \quad \text { and } \\
& w=\left[\left(\left\{u_{\alpha, \beta}^{\prime}\right\},\left\{\left(v_{\alpha}^{\prime}, w_{\alpha}^{\prime}\right)\right\}\right)\right] \in \mathbf{H}^{1}\left(\mathcal{C} \times_{T} \widehat{U}, \mathcal{F}_{\widehat{U}}^{\bullet}\right)
\end{aligned}
$$

we put

$$
\begin{aligned}
& \omega_{1}(v, w)=\left[\left(\left\{\operatorname{Tr}\left(\eta\left(u_{\alpha \beta}\right) \circ \eta\left(u_{\beta \gamma}^{\prime}\right)\right)\right\},-\left\{\operatorname{Tr}\left(\eta\left(u_{\alpha \beta}\right) \circ v_{\beta}^{\prime}\right)-\operatorname{Tr}\left(v_{\alpha} \circ \eta\left(u_{\alpha \beta}^{\prime}\right)\right)\right\}\right)\right] \quad \text { and } \quad(3.5) \\
& \omega_{2}(v, w)=\left[\left\{2 \cdot \operatorname{symb}_{1}\left(u_{\beta \alpha}\right) \circ \operatorname{symb}_{1}\left(u_{\beta \gamma}^{\prime}\right) \circ \psi_{\beta}\right\},-\left\{\operatorname{symb}_{1}\left(u_{\beta \alpha}\right) \circ w_{\beta}^{\prime}+w_{\alpha} \circ \operatorname{symb}_{1}\left(u_{\alpha \beta}^{\prime}\right)\right\}\right] .
\end{aligned}
$$

For each affine open subset $\widehat{U} \subset \widehat{M}$, we define a pairing

$$
\begin{aligned}
& \mathbf{H}^{1}\left(\mathcal{C} \times_{T} \widehat{U}, \mathcal{F}_{\widehat{U}}^{\bullet}\right) \otimes \mathbf{H}^{1}\left(\mathcal{C} \times_{T} \widehat{U}, \mathcal{F}_{\widehat{U}}^{\bullet}\right) \longrightarrow \mathbf{H}^{2}\left(\mathcal{C} \times_{T} \widehat{U}, \Omega_{\mathcal{C} \times_{T} \widehat{U} / \widehat{U}}\right) \cong H^{0}\left(\mathcal{O}_{\widehat{U}}\right), \\
& v \otimes w \longmapsto \omega_{1}(v, w)+\omega_{2}(v, w),
\end{aligned}
$$

where we consider in Čech cohomology with respect to an affine open covering $\left\{U_{\alpha}\right\}$ of $\mathcal{C} \times_{T} U$, $\left\{u_{\alpha \beta}\right\} \in C^{1}\left(\mathcal{F}^{0}\right),\left\{\left(v_{\alpha}, w_{\alpha}\right)\right\} \in C^{0}\left(\mathcal{F}^{1}\right)$ and so on. This pairing determines a pairing

$$
\omega: \mathbf{R}^{1}\left(\pi_{\widehat{M}}\right)_{*}\left(\mathcal{F}^{\bullet}\right) \otimes \mathbf{R}^{1}\left(\pi_{\widehat{M}}\right)_{*}\left(\mathcal{F}^{\bullet}\right) \longrightarrow \mathcal{O}_{\widehat{M}}
$$

By the same argument as in the proof of [15, Proposition 7.2], $\omega$ is skew symmetric and nondegenerate.

Proposition 3.8. For the 2-form constructed in Proposition 3.7, we have $d \omega=0$.

Proof. Let $\Theta_{\widehat{M}_{\nu}}^{\text {initial }}$ be the subbundle of $\Theta_{\widehat{M}_{\nu}}$ consisted by the images of the tangent morphism $\Theta_{\widehat{M}_{\nu} / T^{*} \tilde{M}_{g, n}} \rightarrow \Theta_{\widehat{M}_{\nu}}$ and let $\Theta_{\widehat{M}_{\nu}}^{\mathrm{IMD}}$ be the subbundle of $\Theta_{\widehat{M}_{\nu}}$ consisted by the images of $\widehat{\mathfrak{D}}\left(\hat{\pi}_{\nu}^{*}\left(\Theta_{T^{*} \tilde{M}_{g, n}}\right)\right) \rightarrow \Theta_{\widehat{M}_{\nu}}$. We take an affine open set $\widehat{U} \subset \widehat{M}_{\boldsymbol{\nu}}$. We have a canonical decomposition

$$
\mathbf{H}^{1}\left(\mathcal{F}_{\widehat{U}}^{\bullet}\right) \longrightarrow \Theta_{\widehat{U}}^{\text {initial }} \oplus \Theta_{\widehat{U}}^{\mathrm{IMD}}, \quad v=\left[\left\{u_{\alpha \beta}\right\},\left\{\left(v_{\alpha}, w_{\alpha}\right)\right\}\right] \longmapsto v_{\text {initial }}+v_{\mathrm{IMD}}
$$


where

$$
v_{\text {initial }}=\left[\left\{\eta\left(u_{\alpha \beta}\right)\right\},\left\{\left(v_{\alpha}, 0\right)\right\}\right] \quad \text { and } \quad v_{\mathrm{IMD}}=\left[\left\{\iota(\tilde{\nabla}) \circ \operatorname{symb}_{1}\left(u_{\alpha \beta}\right)\right\},\left\{\left(0, w_{\alpha}\right)\right\}\right] .
$$

We may assume that $\boldsymbol{\nu}$ is generic. Let $\widehat{U}$ be an affine open set of $\widehat{M}_{\boldsymbol{\nu}}$ and let $\left(\tilde{E}, \tilde{\nabla},\left\{\tilde{l}_{j}^{(i)}\right\}, \tilde{\psi}\right)$ be the family on $\mathcal{C} \times_{\tilde{M}_{g, n}} \widehat{U}$. We take an affine open covering $\mathcal{C}_{\widehat{U}}=\bigcup_{\alpha} U_{\alpha}$ such that $\phi_{\alpha}:\left.\tilde{E}\right|_{U_{\alpha}} \stackrel{\sim}{\rightarrow} \mathcal{O}_{U_{\alpha}}^{\oplus r}$ for any $\alpha, \sharp\left\{i\left|\tilde{t}_{i}\right|_{\mathcal{C}_{U}} \cap U_{\alpha} \neq \varnothing\right\} \leq 1$ for any $\alpha$ and $\sharp\left\{\alpha\left|\tilde{t}_{i}\right|_{\mathcal{C}_{U}} \cap U_{\alpha} \neq \varnothing\right\} \leq 1$ for any $i$. If we replace $U_{\alpha}$ sufficiently smaller, there exists a sheaf $E_{\alpha}$ on $U_{\alpha}$ such that $\left.E_{\alpha}\right|_{U_{\alpha} \cap U_{\beta}} \cong$ $\left(\left.\pi_{\widehat{M}_{\nu}}^{-1} \mathcal{O}_{\widehat{M}_{\nu}}\right|_{U_{\alpha} \cap U_{\beta}}\right)^{\oplus r^{2}}$ for any $\beta \neq \alpha$ and an isomorphism $\phi_{\alpha}:\left.j_{*}(\widehat{\boldsymbol{V}})\right|_{U_{\alpha}} \stackrel{\sim}{\rightarrow} E_{\alpha}$. Here the local system $\widehat{\boldsymbol{V}}$ is defined in Section 3.2. For each $\alpha, \beta$, we put

$$
\varphi_{\alpha \beta}:\left.\left.\left.E_{\beta}\right|_{U_{\alpha} \cap U_{\beta}} \stackrel{\phi_{\beta}^{-1}}{\longrightarrow} j_{*}(\widehat{\boldsymbol{V}})\right|_{U_{\alpha} \cap U_{\beta}} \stackrel{\phi_{\alpha}}{\longrightarrow} E_{\alpha}\right|_{U_{\alpha} \cap U_{\beta}} .
$$

For each $\alpha, \beta$, let $\mu_{\alpha \beta}: U_{\alpha \beta} \rightarrow U_{\alpha \beta}$ be an isomorphism such that the glueing scheme of the collection $\left(U_{\alpha}, U_{\alpha \beta}, \mu_{\alpha \beta}\right)$ is isomorphic to $\mathcal{C}_{\widehat{U}}$.

We consider a vector field $v \in H^{0}\left(\widehat{U}, \Theta_{\widehat{U}}\right)$. Then $v$ corresponds to a derivation $D_{v}: \mathcal{O}_{\widehat{U}} \rightarrow \mathcal{O}_{\widehat{U}}$ which naturally induces a morphism

$$
D_{v}: \mathcal{H o m}\left(\left.E_{\beta}\right|_{U_{\alpha} \cap U_{\beta}},\left.E_{\alpha}\right|_{U_{\alpha} \cap U_{\beta}}\right) \longrightarrow \mathcal{H o m}\left(\left.E_{\beta}\right|_{U_{\alpha} \cap U_{\beta}},\left.E_{\alpha}\right|_{U_{\alpha} \cap U_{\beta}}\right) .
$$

The isomorphism $\Theta_{\widehat{M}_{\nu}} \cong \mathbf{R}^{1}\left(\pi_{\widehat{M}_{\nu}}\right)_{*}\left(\left(\widehat{\mathcal{F}}^{\bullet}\right)^{\text {an }}\right)$ is given by

$$
\Theta_{\widehat{M}_{\nu}} \ni v \longmapsto\left[\left\{\left(\phi_{\alpha}^{-1} \circ D_{v}\left(\varphi_{\alpha \beta}\right) \circ \phi_{\beta}, D_{v}\left(\mu_{\alpha \beta}\right)\right)\right\},\left\{D_{v}\left(\left.\psi\right|_{U_{\alpha}}\right)\right\}\right] \in \mathbf{R}^{1}\left(\pi_{\widehat{M}_{\nu}}\right)_{*}\left(\left(\widehat{\mathcal{F}}^{\bullet}\right)^{\text {an }}\right),
$$

and the 2 -form $\omega(u, v)=\omega_{1}(u, v)+\omega_{2}(u, v), u, v \in \Theta_{\widehat{M}}$, is given by

$$
\begin{aligned}
\omega_{1}(u, v)= & {\left[\left\{\operatorname{Tr}\left(D_{u_{\text {initial }}}\left(\varphi_{\alpha \beta}\right) D_{v_{\text {initial }}}\left(\varphi_{\beta \gamma}\right) \varphi_{\gamma \alpha}\right)\right\}\right] \quad \text { and } } \\
\omega_{2}(u, v)=[ & \left\{\left.2 D_{u_{\text {IMD }}}\left(\mu_{\beta \alpha}\right) D_{v_{\text {IMD }}}\left(\mu_{\beta \alpha}\right) \tilde{\psi}_{\beta}\right|_{U_{\alpha \beta}}\right\}, \\
& \left.\left\{-D_{u_{\text {IMD }}}\left(\mu_{\beta \alpha}\right) D_{v_{\text {IMD }}}\left(\left.\tilde{\psi}_{\beta}\right|_{U_{\alpha \beta}}\right)-D_{u_{\text {IMD }}}\left(\left.\tilde{\psi}_{\alpha}\right|_{U_{\alpha \beta}}\right) D_{v_{\text {IMD }}}\left(\mu_{\alpha \beta}\right)\right\}\right] .
\end{aligned}
$$

Since the image of $\Theta_{\widehat{U}}^{\mathrm{IMD}}$ under the tangent morphism of $\widehat{M}_{\boldsymbol{\nu}} \rightarrow M_{\boldsymbol{\nu}}$ determines the foliation determined by the isomonodromic deformations, we can show that

$$
d \omega_{1}(u, v, w)=d \omega_{1}\left(u_{\text {initial }}, v_{\text {initial }}, w_{\text {initial }}\right) .
$$

We have $d \omega_{1}\left(u_{\text {initial }}, v_{\text {initial }}, w_{\text {initial }}\right)=0$ by $[15$, Proposition 7.3$]$. We can also show that $d \omega_{2}(u, v, w)=0$. Then we have the closeness of $\omega=\omega_{1}+\omega_{2}$.

\subsection{Extended phase spaces of isomonodromic deformations}

Proposition 3.9. The morphism $\hat{\pi}_{\nu}: \widehat{M}_{\mathcal{C} / \tilde{M}_{g, n}}^{\alpha}(\tilde{\boldsymbol{t}}, r, e)_{\nu} \rightarrow T^{*} \tilde{M}_{g, n}$ is a Poisson map.

Proof. Let $\hat{\pi}_{\boldsymbol{\nu}}^{t}: \Theta_{\widehat{M}_{\nu}} \rightarrow \pi^{*} \Theta_{T^{*} \tilde{M}_{g, n}}$ be the tangent morphism. We denote by $\tilde{\xi}: \Theta_{T^{*} \tilde{M}_{g, n}} \rightarrow$ $\Omega_{T^{*} \tilde{M}_{g, n}}^{1}$ and $\xi: \Theta_{\widehat{M}_{\nu}} \rightarrow \Omega_{\widehat{M}_{\nu}}^{1}$ the homomorphisms induced by the symplectic structures on $T^{*} \tilde{M}_{g, n}$ and $\widehat{M}_{\nu}$, respectively. The assertion follows from that the following diagram

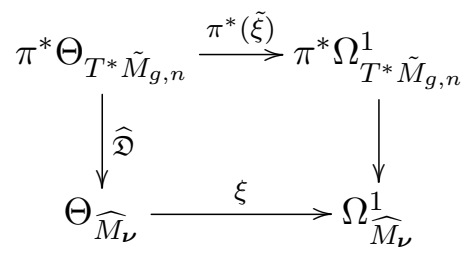

is commutative and $\hat{\pi}_{\nu}^{t} \circ \widehat{\mathfrak{D}}=\mathrm{id}$. Here, $\widehat{\mathfrak{D}}$ is the homomorphism (3.4). 
Let $\mu_{1}, \ldots \mu_{3 g-3+n}$ be local vector fields on an affine open subset $U \subset \tilde{M}_{g, n}$. Let $h_{i}$ be a linear function on $T^{*} \tilde{M}_{g, n}$ corresponding to the local vector field $\mu_{i}$ on $U$. Assume that $\left\{h_{i}, h_{j}\right\}_{\tilde{M}_{g, n}}=0$ for $i, j=1, \ldots, 3 g-3+n$ and $d h_{1} \wedge \cdots \wedge d h_{3 g-3+n}$ is not identically 0 , where $\{\cdot, \cdot\}_{\tilde{M}_{g, n}}$ is the Poisson bracket associated to the symplectic structure $\omega_{\tilde{M}_{g, n}}$. Put $\widehat{U}=\left(\hat{\pi}_{\nu} \circ p_{\tilde{M}_{g, n}}\right)^{-1}(U)$, where $\hat{\pi}_{\boldsymbol{\nu}}: \widehat{M}_{\mathcal{C} / \tilde{M}_{g, n}}^{\boldsymbol{\alpha}}(\tilde{\boldsymbol{t}}, r, e)_{\nu} \rightarrow T^{*} \tilde{M}_{g, n}$ and $p_{\tilde{M}_{g, n}}: T^{*} \tilde{M}_{g, n} \rightarrow \tilde{M}_{g, n}$. Let $\omega_{T^{*} \tilde{M}_{g, n}}$ be the symplectic structure on $T^{*} \tilde{M}_{g, n}$. We define a Hamiltonian $E_{i}$ on $\widehat{U}$ as $\hat{\pi}^{*} h_{i}$ for $i=1, \ldots, 3 g-3+n$. Let $a_{i}(i=1, \ldots, 3 g-3+n)$ be constants. We call the Hamiltonian vector field on $\widehat{U}$ associated to $\sum_{i=1}^{3 g-3+n} a_{i} \mu_{i}$ the vector field $\sum_{i=1}^{3 g-3+n} a_{i}\left\{\cdot, E_{i}\right\}$ on $\widehat{U}$.

Proposition 3.10. First, the Hamiltonians $E_{i}$ satisfy $\left\{E_{i}, E_{j}\right\}=0$ for $i, j=1, \ldots, 3 g-3+n$. In particular, the functions $E_{i}$ are conserved quantities associated to the Hamiltonian vector fields. Second, the restriction of the Hamiltonian vector field associated to $\sum_{i=1}^{3 g-3+n} a_{i} \mu_{i}$ to the common level surface $E_{1}=0, \ldots, E_{3 g-3+n}=0$ in $\widehat{U}$, is coincide with $\mathfrak{D}\left(\sum_{i=1}^{3 g-3+n} a_{i} \mu_{i}\right)$, which is a vector field associated to isomonodromic deformations. Here we consider the vector field $3 g-3+n$ $\sum_{i=1}^{3+n} a_{i} \mu_{i}$ as an element of $\pi_{\nu}^{*}\left(\Theta_{\tilde{M}_{g, n}}\right)(\widehat{U})$.

Proof. Let $\{\cdot, \cdot\}_{\tilde{M}_{g, n}}$ be the Poisson bracket associated to the symplectic structure $\omega_{T^{*} \tilde{M}_{g, n}}$ on $T^{*} \tilde{M}_{g, n}$. Let $v_{h_{i}}$ be the element $\hat{\pi}_{\nu}^{*}\left(\Theta_{T^{*} \tilde{M}_{g, n}}\right)(\widehat{U})$ defined by the vector field $\left\{\cdot, h_{i}\right\}_{\tilde{M}_{g, n}}$ on $\hat{\pi}(\widehat{U}) \subset \tilde{M}_{g, n}$. In other words, $\omega_{T^{*} \tilde{M}_{g, n}}\left(v_{h_{i}}, v\right)=d h_{i}(v)$ for any $v \in \Theta_{U}$. Put $v_{h_{i}}=$ $\left[\left\{d_{\alpha \beta}^{h_{i}}\right\},\left\{w_{\alpha}^{h_{i}}\right\}\right] \in \mathbf{H}^{1}\left(\mathcal{G}_{\widehat{U}}^{\bullet}\right)$, where $\mathcal{G}^{\bullet}$ is the complex (3.3). Put $v_{h_{i}}^{\mathrm{IMD}}=\left[\left\{\iota(\tilde{\nabla})\left(d_{\alpha \beta}^{h_{i}}\right)\right\},\left\{\left(0, w_{\alpha}^{h_{i}}\right)\right\}\right]$ $\in \mathbf{H}^{1}\left(\mathcal{F}_{\widehat{U}}^{\bullet}\right)$. By the diagram (3.6), we have $\omega\left(v_{h_{i}}^{\mathrm{IMD}}, v\right)=d E_{i}(v)$ for any $v \in \Theta_{\widehat{U}}$, that is, $v_{h_{i}}^{\mathrm{IMD}}=\left\{\cdot, E_{i}\right\}$, which is the Hamiltonian vector field associated to $\mu_{i}$.

Note that $\left\{E_{i}, E_{j}\right\}=\omega\left(v_{h_{i}}^{\mathrm{IMD}}, v_{h_{j}}^{\mathrm{IMD}}\right)=\omega_{T^{*} \tilde{M}_{g, n}}\left(v_{h_{i}}, v_{h_{j}}\right)=\left\{h_{i}, h_{j}\right\}_{\tilde{M}_{g, n}}$. By the assumption that the linear functions $h_{i}$ satisfy $\left\{h_{i}, h_{j}\right\}_{\tilde{M}_{g, n}}=0$, we have $\left\{E_{i}, E_{j}\right\}=0$. The common level surface $E_{1}=\cdots=E_{3 g-3+n}=0$ is $M_{\mathcal{C} / \tilde{M}_{g, n}}^{\alpha}(\tilde{\boldsymbol{t}}, r, e)_{\nu}$. On this common level surface, the vector field associated to the Hamiltonian vector field of $\mu_{i}$ is $\left[\left\{\iota(\tilde{\nabla})\left(d_{\alpha \beta}^{h_{i}}\right)\right\},\{0\}\right] \in \mathbf{H}^{1}\left(\mathcal{F}_{\widehat{U}}^{0} \rightarrow \widetilde{\mathcal{F}}_{\widehat{U}}^{1}\right)$, which is a vector field associated to the isomonodromic deformations.

\section{Moduli stack of stable parabolic connections with a quadratic differential and twisted cotangent bundle}

Let $C$ be a smooth projective curve of genus $g, g \geq 2$. The map from the moduli space of pairs $(E, \nabla)$ to the moduli space of vector bundles defined by $(E, \nabla) \mapsto E$ is a twisted cotangent bundle on the moduli space of vector bundles. Here, $E$ is a rank $r$ vector bundle on the fixed curve $C$ and $\nabla$ is a holomorphic connection on $E$. This twisted cotangent bundle has been investigated by Faltings, Ben-Zvi-Biswas, and Ben-Zvi-Frenkel (see [10, Section 4], [8, Section 5], [5, Section 5], and [7, Section 4.1]). Moreover, Ben-Zvi-Biswas and Ben-Zvi-Frenkel studied on a twisted cotangent bundle on the moduli space of pairs $(C, E)$ (see [5, Section 6] and [7, Section 4.3]). In [5, 6], Ben-Zvi and Biswas have introduced extended connections, which are generalization of holomorphic connections. We can define a natural map from the moduli space of extended connections to the moduli space of pairs $(C, E)$. This map is generalization of the map $(E, \nabla) \mapsto E$ and has been investigated in [5, Section 6] and [7, Section 4.3]. In this section, we consider parabolic connections instead of holomorphic connections and study 
the moduli space of parabolic connections with a quadratic differential instead of the moduli space of extended connections. The purpose of this section is to show that the moduli space of parabolic connections with a quadratic differential is equipped with structure of a twisted cotangent bundles. In Section 4.1, we consider the moduli stack corresponding to the moduli scheme considered in the previous section. We introduce the moduli stack of pointed smooth projective curves and quasi-parabolic bundles. We consider the cotangent bundle of this moduli stack. We describe the tangent sheaf of the total space of this cotangent bundle and the canonical symplectic form on this cotangent bundle. In Section 4.2, we consider a map from the moduli stack of parabolic connections with a quadratic differential to the moduli stack of pointed smooth projective curves and quasi-parabolic bundles. We endow this map with structure of a twisted cotangent bundle. In Section 4.3, we introduce extended parabolic connections, which are generalization of parabolic connections and also extended connections. We consider a relation between parabolic connections with a quadratic differential (which are also generalization of parabolic connections) and extended parabolic connections.

In this section, we assume that $\boldsymbol{\nu}$ is generic. If $\boldsymbol{\nu}$ is generic, then any $(\boldsymbol{t}, \boldsymbol{\nu})$-parabolic connection is irreducible. So all $(\boldsymbol{t}, \boldsymbol{\nu})$-parabolic connections are stable.

\subsection{Moduli stack of stable parabolic connections with a quadratic differential}

Let $\mathfrak{M}_{g, n}$ be the moduli stack of $n$-pointed smooth projective curves of genus $g$, where $n$-points consist of distinct points. Let $\widehat{\mathfrak{M}}_{g, n}(r, e, \boldsymbol{\nu})$ be the moduli stack of collections $((C, \boldsymbol{t}, \psi),(E, \nabla, \boldsymbol{l}))$, where $(C, \boldsymbol{t}), \boldsymbol{t}=\left(t_{1}, \ldots, t_{n}\right)$, is an $n$-pointed smooth projective curve of genus $g$ over $\mathbb{C}$ where $t_{1}, \ldots, t_{n}$ are distinct points, $\psi$ is an element of $H^{0}\left(C, \Omega_{C}^{\otimes 2}(D(\boldsymbol{t}))\right)$, and $(E, \nabla, \boldsymbol{l})$ is a $(\boldsymbol{t}, \boldsymbol{\nu})$ parabolic connection of rank $r$ and of degree $e$ on $C$. Let $\Theta_{\widehat{\mathfrak{M}}_{g, n}(r, e, \boldsymbol{\nu})}$ be the tangent complex of $\widehat{\mathfrak{M}}_{g, n}(r, e, \boldsymbol{\nu})$, that is, for each smooth map $f_{U}: U \rightarrow \widehat{\mathfrak{M}}_{g, n}(r, e, \boldsymbol{\nu})$ from a scheme $U$, the pull-back $f_{U}^{*} \Theta_{\widehat{\mathfrak{M}}_{g, n}(r, e, \boldsymbol{\nu})}$ is $\Theta_{U / \widehat{\mathfrak{M}}_{g, n}(r, e, \boldsymbol{\nu})} \rightarrow \Theta_{U}$ considered as a length 2 complex supported in degree -1 and 0 . Here $\Theta_{U / \widehat{\mathfrak{M}}_{g, n}(r, e, \boldsymbol{\nu})}:=\Delta^{*}\left(\Theta_{\left(U \times_{\widehat{\mathfrak{M}}_{g, n}(r, e, \boldsymbol{\nu})} U\right) / U}\right)$, where $U \rightarrow U \times_{\widehat{\mathfrak{M}}_{g, n}(r, e, \boldsymbol{\nu})} U$ is the diagonal. Let $\Theta_{\widehat{\mathfrak{M}}_{g, n}(r, e, \boldsymbol{\nu}), x}$ be the fiber of $\Theta_{\widehat{\mathfrak{M}}_{g, n}(r, e, \boldsymbol{\nu})}$ over a point $x=((C, \boldsymbol{t}, \psi),(E, \nabla, \boldsymbol{l}))$ of $\widehat{\mathfrak{M}}_{g, n}(r, e, \boldsymbol{\nu})$. Then $H^{0}\left(\Theta_{\widehat{\mathfrak{M}}_{g, n}(r, e, \boldsymbol{\nu}), x}\right)$ is isomorphic to $\mathbf{H}^{1}\left(\mathcal{F}_{x}^{\bullet}\right)$. Here, we recall the complex $\mathcal{F}_{x}^{\bullet}$ :

$$
\begin{aligned}
\mathcal{F}_{x}^{0} & :=\left\{s \in \mathcal{A}_{E}(D(\boldsymbol{t}))\left|\left(s-\iota(\nabla) \circ \operatorname{symb}_{1}(s)\right)\right|_{t_{i}}\left(l_{j}^{(i)}\right) \subset l_{j}^{(i)} \text { for any } i, j\right\}, \\
\widetilde{\mathcal{F}}_{x}^{1} & :=\left\{s \in \mathcal{E}_{\operatorname{nd}}(E) \otimes \Omega_{C}^{1}(D(\boldsymbol{t})) \mid \operatorname{res}_{t_{i}}(s)\left(l_{j}^{(i)}\right) \subset l_{j+1}^{(i)} \text { for any } i, j\right\}, \\
\mathcal{F}_{x}^{1} & :=\widetilde{\mathcal{F}}_{x}^{1} \oplus \Omega_{C}^{\otimes 2}(D(\boldsymbol{t})) ; \text { and } d_{\mathcal{F}} \bullet:=\left(d_{\nabla}, d_{\psi}\right) \circ\left(\operatorname{Id}-\iota(\tilde{\nabla}) \circ \operatorname{symb}_{1}, \operatorname{symb}_{1}\right): \mathcal{F}_{x}^{0} \longrightarrow \mathcal{F}_{x}^{1},
\end{aligned}
$$

where $d_{\nabla}: \widetilde{\mathcal{F}}^{0} \rightarrow \widetilde{\mathcal{F}}^{1}, s \mapsto \nabla \circ s-s \circ \nabla$ and $d_{\psi}: \Theta_{C}(-D(\boldsymbol{t})) \rightarrow \Omega_{C}^{\otimes 2}(D(\boldsymbol{t}))$ defined by (3.1). The pairing $\mathbf{H}^{1}\left(\mathcal{F}_{x}^{\bullet}\right) \otimes \mathbf{H}^{1}\left(\mathcal{F}_{x}^{\bullet}\right) \rightarrow \mathbf{H}^{2}\left(\Omega_{C}^{\bullet}\right)$ defined by (3.5) gives a symplectic structure on $\widehat{\mathfrak{M}}_{g, n}(r, e, \nu)$.

Definition 4.1. Let $(C, \boldsymbol{t})$ be an $n$-pointed smooth projective curve of genus $g$ over $\mathbb{C}$ where $t_{1}, \ldots, t_{n}$ are distinct points. We say $(E, \boldsymbol{l}), \boldsymbol{l}=\left\{l_{*}^{(i)}\right\}_{1 \leq i \leq n}$, is a quasi-parabolic bundle of rank $r$ and of degree $e$ on $(C, t)$ if $E$ is a rank $r$ algebraic vector bundle of degree $e$ on $C$, and for each $t_{i}, l_{*}^{(i)}$ is a filtration $\left.E\right|_{t_{i}}=l_{0}^{(i)} \supset l_{1}^{(i)} \supset \cdots \supset l_{r}^{(i)}=0 \operatorname{such}$ that $\operatorname{dim}\left(l_{j}^{(i)} / l_{j+1}^{(i)}\right)=1$, $j=0,1, \ldots, r-1$.

Let $\mathfrak{P}_{g, n}(r, e)$ be the moduli stack of pairs $((C, \boldsymbol{t}),(E, \boldsymbol{l}))$, where $(C, \boldsymbol{t})\left(\boldsymbol{t}=\left(t_{1}, \ldots, t_{n}\right)\right)$ is an $n$-pointed smooth projective curve of genus $g$ over $\mathbb{C}$ where $t_{1}, \ldots, t_{n}$ are distinct points, and $(E, \boldsymbol{l})$ is a quasi-parabolic bundle of rank $r$ and of degree $e$ on $(C, \boldsymbol{t})$. We have a projection $\mathfrak{P}_{g, n}(r, e) \rightarrow \mathfrak{M}_{g, n}$. Let $\mathfrak{P}_{g, n}(r, e, \boldsymbol{\nu})$ be the substack defined by the condition where a quasiparabolic bundle admits a $(\boldsymbol{t}, \boldsymbol{\nu})$-parabolic connection. Let $\pi_{\mathfrak{P}_{g, n}(r, e, \boldsymbol{\nu})}$ and $\pi_{\mathfrak{M}_{g, n}}$ be the following 
morphisms:

$$
\begin{aligned}
& \pi_{\mathfrak{P}_{g, n}(r, e, \boldsymbol{\nu})}: \widehat{\mathfrak{M}}_{g, n}(r, e, \boldsymbol{\nu}) \longrightarrow \mathfrak{P}_{g, n}(r, e, \boldsymbol{\nu}), \quad((C, \boldsymbol{t}, \psi),(E, \nabla, \boldsymbol{l})) \longmapsto((C, \boldsymbol{t}),(E, \boldsymbol{l})), \\
& \pi_{\mathfrak{M}_{g, n}}: \mathfrak{P}_{g, n}(r, e, \boldsymbol{\nu}) \longrightarrow \mathfrak{M}_{g, n}, \quad((C, \boldsymbol{t}),(E, \boldsymbol{l})) \longmapsto(C, \boldsymbol{t}) .
\end{aligned}
$$

Let $\Theta_{\mathfrak{P}_{g, n}(r, e, \boldsymbol{\nu})}$ be the tangent complex of $\mathfrak{P}_{g, n}(r, e, \boldsymbol{\nu})$. Let $\Theta_{\mathfrak{P}_{g, n}(r, e, \boldsymbol{\nu}), p}$ be the fiber of $\Theta_{\mathfrak{P}_{g, n}(r, e, \boldsymbol{\nu})}$ over a point $p=((C, \boldsymbol{t}),(E, \boldsymbol{l}))$ of $\mathfrak{P}_{g, n}(r, e, \boldsymbol{\nu})$.

We consider infinitesimal deformations of $p=((C, \boldsymbol{t}),(E, \boldsymbol{l}))$. We put

$$
\begin{aligned}
& \widetilde{\mathcal{H}}_{p}^{0}:=\left\{s \in \mathcal{E} \operatorname{nd}(E)|s|_{t_{i}}\left(l_{j}^{(i)}\right) \subset l_{j}^{(i)} \text { for any } i, j\right\} \quad \text { and } \\
& \widetilde{\mathcal{H}}_{p}^{1}:=\left\{s \in \mathcal{E} \operatorname{nd}(E) \otimes \Omega_{C}^{1}(D(\boldsymbol{t})) \mid \operatorname{res}_{t_{i}}(s)\left(l_{j}^{(i)}\right) \subset l_{j+1}^{(i)} \text { for any } i, j\right\} .
\end{aligned}
$$

Note that $\left(\widetilde{\mathcal{H}}_{p}^{0}\right)^{*} \otimes \Omega_{C}^{1} \cong \widetilde{\mathcal{H}}_{p}^{1}$. Put

$$
\mathcal{H}_{p}^{0}:=\left\{s \in \mathcal{A}_{E}(D(\boldsymbol{t})) \subset \mathcal{E}_{\operatorname{nd}_{\mathbb{C}}}(E)|s|_{t_{i}}\left(l_{j}^{(i)}\right) \subset l_{j}^{(i)} \text { for any } i, j\right\}
$$

and $\mathcal{H}_{p}^{1}:=\left(\mathcal{H}_{p}^{0}\right)^{*} \otimes \Omega_{C}^{1}$. Then we have exact sequences

$$
\begin{aligned}
& 0 \longrightarrow \widetilde{\mathcal{H}}_{p}^{0} \longrightarrow \mathcal{H}_{p}^{0} \stackrel{\text { symb }_{1}}{\longrightarrow} \Theta_{C}(-D(\boldsymbol{t})) \longrightarrow 0 \quad \text { and } \\
& 0 \longrightarrow \Omega_{C}^{\otimes 2}(D(\boldsymbol{t})) \stackrel{q}{\longrightarrow} \mathcal{H}_{p}^{1} \stackrel{\kappa}{\longrightarrow} \widetilde{\mathcal{H}}_{p}^{1} \longrightarrow 0
\end{aligned}
$$

We take an affine open covering $\left\{U_{i}\right\}$ of $C$ so that we can take a trivialization $\phi_{i}:\left.E\right|_{U_{i}} \cong \mathcal{O}_{U_{i}}^{\oplus r}$ of $E$ on each $U_{i}$ and the restriction of $\mathcal{H}_{p}^{1}$ to $U_{i}$ is $\mathcal{O}_{U_{i}}$-isomorphic to the direct $\operatorname{sum}\left(\widetilde{\mathcal{H}}_{p}^{1}\right)_{U_{i}} \oplus$ $\Omega_{C}^{\otimes 2}(D(\boldsymbol{t}))_{U_{i}}$. We fix trivializations of $E$. On $U_{i} \cap U_{i}$, the transformation $\left(\mathcal{H}_{p}^{1}\right)_{U_{i}} \rightarrow\left(\mathcal{H}_{p}^{1}\right)_{U_{j}}$ is given by

$$
\begin{aligned}
& \left(\Phi_{i}\left(f_{i}\right) d f_{i}, \phi_{i}\left(f_{i}\right) d f_{i} \otimes d f_{i}\right) \longmapsto\left(\Phi_{j}\left(f_{i}\right) d f_{i}, \phi_{j}\left(f_{i}\right) d f_{i} \otimes d f_{i}\right) \\
& \quad:=\left(\left(\theta_{i j}^{-1} \Phi_{i}\left(f_{i}\right) \theta_{i j}\right) d f_{i}, \phi_{i}\left(f_{i}\right) d f_{i} \otimes d f_{i}+\operatorname{Tr}\left(\theta_{i j}^{-1} \Phi_{i}\left(f_{i}\right) \frac{\partial \theta_{i j}}{\partial f_{i}}\right) d f_{i} \otimes d f_{i}\right),
\end{aligned}
$$

where $\theta_{i j}:=\phi_{i} \circ \phi_{j}^{-1}: \mathcal{O}_{U_{i} \cap U_{j}}^{\oplus r} \rightarrow \mathcal{O}_{U_{i} \cap U_{j}}^{\oplus r}$ is a transition function of $E$. Then $H^{0}\left(\Theta_{\mathfrak{P}_{g, n}(r, e, \boldsymbol{\nu}), p}\right)$ is isomorphic to $H^{1}\left(\mathcal{H}_{p}^{0}\right)$, and $H^{0}\left(\mathcal{H}_{p}^{1}\right)$ is the dual of $H^{1}\left(\mathcal{H}_{p}^{0}\right)$. The vector space $H^{0}\left(\mathcal{H}_{p}^{1}\right)$ is the space of 1 -forms at $p$.

Put $p=((C, \boldsymbol{t}),(E, \boldsymbol{l}))$. Let $\widehat{\Phi}_{p}$ be an element of $H^{0}\left(\mathcal{H}_{p}^{1}\right)$, which is described by $\left(\Phi_{p}, \phi_{p}\right)$ locally, where $\Phi_{p} d f \in \widetilde{\mathcal{H}}_{p}^{1}$ and $\phi_{p} d f \otimes d f \in \Omega_{C}^{\otimes 2}(D(\boldsymbol{t}))$. We consider infinitesimal deformations of $\left(p, \widehat{\Phi}_{p}\right)$. For $\widehat{\Phi}_{p}$, we define a complex $d^{0}\left(\widehat{\Phi}_{p}\right): \mathcal{H}_{p}^{0} \rightarrow \mathcal{H}_{p}^{1}$ as follows. For each affine open set $U \subset C$, we define the image of $a_{U} \partial / \partial f_{U}+\eta_{U} \in \mathcal{H}_{p}^{0}(U)$ as

$$
\begin{aligned}
& \left(\Phi_{p} d f_{U} \circ \eta_{U}-\eta_{U} \circ \Phi_{p} d f_{U}-\frac{\partial\left(a_{U} \Phi_{p}\right)}{\partial f_{U}} d f_{U},\right. \\
& \left.\operatorname{Tr}\left(\frac{\partial \eta_{U}}{\partial f_{U}} \Phi_{p} d f_{U} \otimes d f_{U}\right)-a_{U} \frac{\partial \phi_{p}}{\partial f_{U}} d f_{U} \otimes d f_{U}-2 \frac{\partial a_{U}}{\partial f_{U}} \phi_{p} d f_{U} \otimes d f_{U}\right) .
\end{aligned}
$$

We can show that this homomorphism on each $U$ gives a homomorphism $d^{0}\left(\widehat{\Phi}_{p}\right): \mathcal{H}_{p}^{0} \rightarrow \mathcal{H}_{p}^{1}$. We consider the first hypercohomology $\mathbf{H}^{1}\left(\mathcal{H}_{p}^{\bullet}\right)$ of $d^{0}\left(\widehat{\Phi}_{p}\right): \mathcal{H}_{p}^{0} \rightarrow \mathcal{H}_{p}^{1}$. By the Čech cohomology, an element of $\mathbf{H}^{1}\left(\mathcal{H}_{p}^{\bullet}\right)$ is described by $\left[\left\{a_{i j} \partial / \partial f_{i}+\eta_{i j}\right\},\left\{\left(\hat{v}_{i}, \hat{w}_{i}\right)\right\}\right]$, where

$$
a_{j k} \frac{\partial f_{i}}{\partial f_{j}}-a_{i k}+a_{i j}=0
$$




$$
\begin{aligned}
& \left(\theta_{j i}^{-1} \eta_{j k} \theta_{j i}+a_{j k} \theta_{j i}^{-1} \frac{\partial \theta_{j i}}{\partial f_{j}}\right)-\eta_{i k}+\eta_{i j}=0, \quad \text { and } \\
& \left(\theta_{j i}^{-1} \hat{v}_{j} \theta_{j i}, \hat{w}_{j}+\operatorname{Tr}\left(\theta_{j i}^{-1} \hat{v}_{j} \frac{\partial \theta_{j i}}{\partial f_{j}} d f_{j}\right)\right)-\left(\hat{v}_{i}, \hat{w}_{i}\right)=d^{0}\left(\widehat{\Phi}_{p}\right)\left(\eta_{i j}\right)
\end{aligned}
$$

for some affine open covering $\left\{U_{i}\right\}_{i}$ of $C$. Infinitesimal deformations of $\left(p, \widehat{\Phi}_{p}\right)$ are parametrized by $\mathbf{H}^{1}\left(\mathcal{H}_{p}^{\bullet}\right)$. Then the fiber of the tangent sheaf of the moduli stack of pairs $(((C, \boldsymbol{t}),(E, \boldsymbol{l})), \widehat{\Phi})$ (where $\left.\widehat{\Phi} \in H^{0}\left(\mathcal{H}_{p}^{1}\right)\right)$ at a point $\left(p, \widehat{\Phi}_{p}\right)$ is isomorphic to $\mathbf{H}^{1}\left(\mathcal{H}_{p}^{\bullet}\right)$. Moreover, we define a paring $\mathbf{H}^{1}\left(\mathcal{H}_{p}^{\bullet}\right) \otimes \mathbf{H}^{1}\left(\mathcal{H}_{p}^{\bullet}\right) \rightarrow \mathbf{H}^{2}\left(\Omega_{C}^{\bullet}\right)$ by

$$
\begin{gathered}
{\left[\left(\left\{a_{i j} \partial / \partial f_{i}+\eta_{i j}\right\},\left\{\left(\hat{v}_{i}, \hat{w}_{i}\right)\right\}\right)\right] \otimes\left[\left(\left\{a_{i j}^{\prime} \partial / \partial f_{i}+\eta_{i j}^{\prime}\right\},\left\{\left(\hat{v}_{i}^{\prime}, \hat{w}_{i}^{\prime}\right)\right\}\right)\right]} \\
\longmapsto \\
\longmapsto\left(\left\{\operatorname{Tr}\left(\eta_{j i}\left(a_{j k}^{\prime} \Phi_{j}\right)\right)+\operatorname{Tr}\left(\left(a_{j i} \Phi_{j}\right) \eta_{j k}^{\prime}\right)-2 a_{j i} a_{j k}^{\prime} \phi_{j}\right\},\right. \\
\left.\left.-\left\{-\operatorname{Tr}\left(\eta_{j i} \hat{v}_{j}^{\prime}\right)+\left(a_{j i} \hat{w}_{j}^{\prime}\right)-\operatorname{Tr}\left(\hat{v}_{i} \eta_{i j}^{\prime}\right)+\left(\hat{w}_{i} a_{i j}^{\prime}\right)\right\}\right)\right] .
\end{gathered}
$$

Proposition 4.2. This pairing gives a symplectic structure on the moduli stack of pairs $(((C, \boldsymbol{t})$, $(E, \boldsymbol{l})), \widehat{\Phi})$.

Proof. We define a 1 -form $\theta_{\mathfrak{P}_{g, n}(r, e, \boldsymbol{\nu})}$ by

$$
\begin{aligned}
& \theta_{\mathfrak{P}_{g, n}(r, e, \boldsymbol{\nu})}: \mathbf{H}^{1}\left(\mathcal{H}_{p}^{\bullet}\right) \longrightarrow H^{1}\left(\Omega_{C}^{1}\right), \\
& {\left[\left\{a_{i j} \partial / \partial f_{i}+\eta_{i j}\right\},\left\{\left(\hat{v}_{i}, \hat{w}_{i}\right)\right\}\right] \longmapsto\left[\operatorname{Tr}\left(\eta_{i j} \Phi_{i} d f_{i}\right)+a_{j i} \phi_{i}\left(\frac{\partial \mu_{i j}}{\partial f_{i}} d f_{j}\right)\right]}
\end{aligned}
$$

for each $\left(\Phi_{i} d f_{i}, \phi_{i} d f_{i} \otimes d f_{i}\right)$. This 1-form $\theta_{\mathfrak{P}_{q, n}(r, e, \boldsymbol{\nu})}$ is the canonical 1-form on the cotangent bundle of $\mathfrak{P}_{g, n}(r, e, \boldsymbol{\nu})$. Let $d \theta_{\mathfrak{P}_{g, n}(r, e, \boldsymbol{\nu})}$ be the exterior differential of $\theta_{\mathfrak{P}_{g, n}(r, e, \boldsymbol{\nu})}$. The 2-form $d \theta_{\mathfrak{P}_{g, n}(r, e, \boldsymbol{\nu})}$ gives the symplectic form on the cotangent bundle of $\mathfrak{P}_{g, n}(r, e, \boldsymbol{\nu})$. We compute the 2-form $d \theta_{\mathfrak{P}_{g, n}(r, e, \boldsymbol{\nu})}$ as follows

$$
\begin{aligned}
\operatorname{Tr}\left(D_{v^{\prime}}\right. & \left.\left(\theta_{i j}\right) D_{v}\left(\theta_{i j}^{-1} \Phi_{i} d f_{i}\right)-D_{v}\left(\theta_{i j}\right) D_{v^{\prime}}\left(\theta_{i j}^{-1} \Phi_{i} d f_{i}\right)\right)+d\left(a_{i j} \operatorname{Tr}\left(\eta_{i j}^{\prime} \Phi_{i}\right)\right) \\
= & \operatorname{Tr}\left(-D_{v^{\prime}}\left(\theta_{i j}\right) \theta_{i j}^{-1} D_{v}\left(\theta_{i j}\right) \theta_{i j}^{-1} \Phi_{i} d f_{i}+D_{v}\left(\theta_{i j}\right) \theta_{i j}^{-1} D_{v^{\prime}}\left(\theta_{i j}\right) \theta_{i j}^{-1} \Phi_{i} d f_{i}\right. \\
& \left.+D_{v^{\prime}}\left(\theta_{i j}\right) \theta_{i j}^{-1} D_{v}\left(\Phi_{i} d f_{i}\right)-D_{v}\left(\theta_{i j}\right) \theta_{i j}^{-1} D_{v^{\prime}}\left(\Phi_{i} d f_{i}\right)\right)+d\left(a_{i j} \operatorname{Tr}\left(\eta_{i j}^{\prime} \Phi_{i}\right)\right) \\
= & \operatorname{Tr}\left(-\eta_{i j}\left(\left[\Phi_{i} d f_{i}, \eta_{i j}^{\prime}\right]-d\left(a_{i j} \Phi_{i}\right)\right)-\eta_{i j} \hat{v}_{i}^{\prime}+\eta_{i j}^{\prime} \hat{v}_{i}\right)+a_{i j} \operatorname{Tr}\left(d\left(\eta_{i j}^{\prime}\right) \Phi_{i}\right) \\
= & -\operatorname{Tr}\left(\eta_{i j} \hat{v}_{j}^{\prime}\right)+\operatorname{Tr}\left(\eta_{i j}^{\prime} \hat{v}_{i}\right)+a_{i j} \operatorname{Tr}\left(d\left(\eta_{i j}^{\prime}\right) \Phi_{i}\right) \\
= & \operatorname{Tr}\left(\eta_{j i} \hat{v}_{j}^{\prime}\right)+\operatorname{Tr}\left(\eta_{i j}^{\prime} \hat{v}_{i}\right)-a_{i j} \operatorname{Tr}\left(\theta_{j i}^{-1} \hat{v}_{j}^{\prime} \frac{\partial \theta_{j i}}{\partial f_{i}}\right)+a_{i j} \operatorname{Tr}\left(d\left(\eta_{i j}^{\prime}\right) \Phi_{i}\right)
\end{aligned}
$$

and

$$
\begin{gathered}
D_{v^{\prime}}\left(\mu_{j i}\right) D_{v}\left(\phi_{i} \frac{\partial \mu_{i j}}{\partial f_{j}}\right) d f_{i}-D_{v}\left(\mu_{j i}\right) D_{v^{\prime}}\left(\phi_{i} \frac{\partial \mu_{i j}}{\partial f_{j}}\right) d f_{i}+d\left(a_{j i}^{\prime} a_{j i} \phi_{j}\right) \\
=a_{i j}\left(-a_{i j}^{\prime} d \psi_{i}-2 \phi_{i} \frac{\partial a_{i j}^{\prime}}{\partial f_{i}} d f_{i}\right)-a_{i j}^{\prime} \hat{w}_{i} d f_{i}+a_{i j} \hat{w}_{i}^{\prime} d f_{i} \\
=a_{i j} \hat{w}_{j}^{\prime} d f_{j}-a_{i j}^{\prime} \hat{w}_{i} d f_{i}+a_{i j} \operatorname{Tr}\left(\theta_{j i}^{-1} \hat{v}_{j}^{\prime} \frac{\partial \theta_{j i}}{\partial f_{i}}\right)-a_{i j} \operatorname{Tr}\left(d\left(\eta_{i j}^{\prime}\right) \Phi_{i}\right) .
\end{gathered}
$$

Then we have this proposition.

Put $p=((C, \boldsymbol{t}),(E, \boldsymbol{l}))$. Let $\nabla$ be a connection: $\nabla: E \rightarrow E \otimes \Omega_{C}^{1}(D(\boldsymbol{t}))$. For a connection $\nabla$, we define a decomposition of $H^{0}\left(\mathcal{H}_{p}^{1}\right)$ as follows

$$
\begin{aligned}
& H^{0}\left(\mathcal{H}_{p}^{1}\right) \longrightarrow H^{0}\left(\widetilde{\mathcal{H}}_{p}^{1}\right) \oplus H^{0}\left(\Omega_{C}^{\otimes 2}(D(\boldsymbol{t}))\right), \\
& \widehat{\Phi} \longmapsto(\kappa(\widehat{\Phi}), \widehat{\Phi}-\psi(\nabla, \kappa(\widehat{\Phi}))) .
\end{aligned}
$$


Here $\psi(\nabla, \kappa(\widehat{\Phi})) \in H^{0}\left(\mathcal{H}_{p}^{1}\right)$ is defined as follows. We take an affine open covering $\left\{U_{i}\right\}$ of $C$ such that on $U_{i}$ the connection $\left.\nabla\right|_{U_{i}}$ is described by $d+A_{i} d f_{i}$ and the Higgs field $\left.\kappa(\widehat{\Phi})\right|_{U_{i}}$ is described by $\Phi_{i} d f_{i}$. On each $U_{i}$, we define an element $\left.\psi(\nabla, \kappa(\widehat{\Phi}))\right|_{U_{i}}$ as

$$
\left.\psi(\nabla, \kappa(\widehat{\Phi}))\right|_{U_{i}}=\left(\Phi_{i} d f_{i}, \operatorname{Tr}\left(\Phi_{i} A_{i}+\frac{1}{2} \Phi_{i} \Phi_{i}\right) d f_{i} \otimes d f_{i}\right) \in \mathcal{H}_{p}^{1}\left(U_{i}\right),
$$

which gives an element $\psi(\nabla, \kappa(\widehat{\Phi})) \in H^{0}\left(\mathcal{H}_{p}^{1}\right)$.

\subsection{Moduli stack as twisted cotangent bundle}

Let $\Gamma\left(\pi_{\mathfrak{P}_{g, n}(r, e, \boldsymbol{\nu})}\right)$ be the sheaf of set on $\mathfrak{P}_{g, n}(r, e, \boldsymbol{\nu})$ where $\Gamma\left(\pi_{\mathfrak{P}_{g, n}(r, e, \boldsymbol{\nu})}\right)(U)$ is the set of sections of $\pi_{\mathfrak{P}_{g, n}(r, e, \boldsymbol{\nu})}$ over $U$ for each smooth map $U \rightarrow \mathfrak{P}_{g, n}(r, e, \boldsymbol{\nu})$. Here $U$ is a scheme. We take a section $\sigma$ and put $\sigma(p)=\left(\nabla_{p}, \psi_{p}\right)$, where $\nabla_{p}: E \rightarrow E \otimes \Omega_{C}^{1}(D(\boldsymbol{t}))$ is a connection such that $\left(E, \boldsymbol{l}, \nabla_{p}\right)$ is a $(\boldsymbol{t}, \boldsymbol{\nu})$-parabolic connection of rank $r$ and of degree $e$ on $C$, and $\psi_{p} \in H^{0}\left(C, \Omega_{C}^{\otimes 2}(D(\boldsymbol{t}))\right)$ for $p=((C, \boldsymbol{t}),(E, \boldsymbol{l}))$.

Definition 4.3. For a 1 -form $\widehat{\Phi}$ on $\mathfrak{P}_{g, n}(r, e, \boldsymbol{\nu})$, we define a translation by

$$
\begin{aligned}
& \Gamma\left(\pi_{\mathfrak{P}_{g, n}(r, e, \boldsymbol{\nu})}\right) \longrightarrow \Gamma\left(\pi_{\mathfrak{P}_{g, n}(r, e, \boldsymbol{\nu})}\right), \\
& \sigma(p)=\left(\nabla_{p}, \psi_{p}\right) \longmapsto t_{\widehat{\Phi}}(\sigma)(p):=\left(\nabla_{p}+\kappa\left(\widehat{\Phi}_{p}\right), \psi_{p}+\left(\widehat{\Phi}_{p}-\psi\left(\nabla_{p}, \kappa\left(\widehat{\Phi}_{p}\right)\right)\right)\right) .
\end{aligned}
$$

By this translation (4.2), we have an $\Omega_{\mathfrak{P}_{g, n}(r, e, \boldsymbol{\nu})}^{1}$-torsor structure on $\Gamma\left(\pi_{\mathfrak{P}_{g, n}(r, e, \boldsymbol{\nu})}\right)$.

Theorem 4.4. Assume that $\boldsymbol{\nu}$ is generic. Let $\omega$ be the symplectic form on $\widehat{\mathfrak{M}}_{g, n}(r, e, \boldsymbol{\nu})$. We define a map c: $\Gamma\left(\pi_{\mathfrak{P}_{g, n}(r, e, \boldsymbol{\nu})}\right) \rightarrow \Omega_{\mathfrak{P}_{g, n}(r, e, \boldsymbol{\nu})}^{2 \mathrm{cl}}$ by $c(\gamma)=\gamma^{*}(\omega)$ for $\gamma \in \Gamma\left(\pi_{\mathfrak{P}_{g, n}(r, e, \boldsymbol{\nu})}\right)$. Then for any $\widehat{\Phi} \in \Omega_{\mathfrak{P}_{g, n}(r, e, \boldsymbol{\nu})}^{1}$ we have $c\left(t_{\widehat{\Phi}}(\gamma)\right)=d(\widehat{\Phi})+c(\gamma)$. That is, $\left(\Gamma\left(\pi_{\mathfrak{P}_{g, n}(r, e, \boldsymbol{\nu})}\right)\right.$, c) is an $\Omega_{\mathfrak{P}_{g, n}(r, e, \nu)}^{\geq \text {torsor. }}$

By this theorem and the argument as in Section 2.3, the morphism $\pi_{\mathfrak{P}_{g, n}(r, e, \boldsymbol{\nu})}: \widehat{\mathfrak{M}}_{g, n}(r, e, \boldsymbol{\nu})$ $\rightarrow \mathfrak{P}_{g, n}(r, e, \boldsymbol{\nu})$ is equipped with structure of a twisted cotangent bundle.

Proof. Put $p=((C, \boldsymbol{t}),(E, \boldsymbol{l}))$. Let $v$ be an element of $H^{1}\left(\mathcal{H}_{p}^{0}\right)$. We take a section $\sigma$ and put $\sigma(p)=\left(\nabla_{p}, \psi_{p}\right)$, where $\nabla_{p}: E \rightarrow E \otimes \Omega_{C}^{1}(D(\boldsymbol{t}))$ is a connection and $\psi_{p} \in H^{0}\left(C, \Omega_{C}^{\otimes 2}(D(\boldsymbol{t}))\right)$.

We take an affine open covering $\left\{U_{i}\right\}$ of $C$ such that elements of $H^{1}\left(\mathcal{H}_{p}^{0}\right)$ are described by the Čech cohomology: $v=\left[\left\{a_{i j} \partial / \partial f_{i}+\eta_{i j}\right\}\right]$, where $a_{i j} \partial / \partial f_{i}+\eta_{i j} \in \mathcal{H}_{p}^{0}\left(U_{i} \cap U_{j}\right)$. Let $\nabla_{p}(v, \epsilon)$, $\widehat{\Phi}_{p}(v, \epsilon)$, and $\psi_{p}(v, \epsilon)$ be the infinitesimal deformations of $\nabla_{p}, \widehat{\Phi}_{p}$, and $\psi_{p}$ associated to $v$ over Spec $\mathbb{C}[\epsilon]$, respectively, where $\epsilon^{2}=0$. We take local descriptions of the connection, the Higgs field, and the quadratic differentials on $U_{i}$ as follows. The connection $\nabla_{p}(v, \epsilon)$ and the Higgs field $\kappa\left(\widehat{\Phi}_{p}\right)(v, \epsilon)$ are described as $d+A_{i} d f_{i}+\epsilon v_{i} \bmod d \epsilon$ and $\Phi_{i} d f_{i}+\epsilon \hat{v}_{i} \bmod d \epsilon$ on $U_{i}$, respectively. Moreover, on $U_{i}$ the quadratic differentials $\psi_{p}(v, \epsilon)$ and $\left(\widehat{\Phi}_{p}-\psi\left(\nabla_{p}, \kappa\left(\widehat{\Phi}_{p}\right)\right)\right)(v, \epsilon)$ are described by $\psi_{i} d f_{i} \otimes d f_{i}+\epsilon w_{i} d f_{i} \otimes d f_{i}$ and $\phi_{i} d f_{i} \otimes d f_{i}+\epsilon \hat{w}_{i} d f_{i} \otimes d f_{i} \bmod d \epsilon$ on $U_{i}$, respectively.

We decompose $\widehat{\Phi}_{p}=\widehat{\Phi}_{1}+\widehat{\Phi}_{2}$, where $\widehat{\Phi}_{1}=\psi\left(\nabla_{p}, \widehat{\Phi}_{p}\right)$ and $\widehat{\Phi}_{2}=\widehat{\Phi}_{p}-\psi\left(\nabla_{p}, \widehat{\Phi}_{p}\right)$. Then we can compute the exterior differentials of $\widehat{\Phi}_{1}$ and $\widehat{\Phi}_{2}$ as follows

$$
\begin{aligned}
d \widehat{\Phi}_{1}\left(v, v^{\prime}\right)= & {\left[\left(\left\{\operatorname{Tr}\left(\eta_{j i}\left(a_{j k}^{\prime} \Phi_{j}\right)\right)+\operatorname{Tr}\left(\left(a_{j i} \Phi_{j}\right) \eta_{j k}^{\prime}\right)-\operatorname{Tr}\left(2 a_{j i} a_{j k}^{\prime} A_{j} \Phi_{j}+a_{j i} a_{j k}^{\prime} \Phi_{j} \Phi_{j}\right)\right\}\right.\right.} \\
& -\left\{-\operatorname{Tr}\left(\eta_{j i} \hat{v}_{j}^{\prime}\right)+\operatorname{Tr}\left(\left(a_{j i} A_{j}\right) \hat{v}_{j}^{\prime}+\left(a_{j i} \Phi_{j}\right) v_{j}^{\prime}+\left(a_{j i} \Phi_{j}\right) \hat{v}_{j}^{\prime}\right)\right. \\
& \left.\left.\left.-\operatorname{Tr}\left(\hat{v}_{i} \eta_{i j}^{\prime}\right)+\operatorname{Tr}\left(\left(a_{i j}^{\prime} A_{i}\right) \hat{v}_{i}+\left(a_{i j}^{\prime} \Phi_{i}\right) v_{i}+\left(a_{i j}^{\prime} \Phi_{i}\right) \hat{v}_{i}\right)\right\}\right)\right], \\
d \widehat{\Phi}_{2}\left(v, v^{\prime}\right)= & {\left[\left(\left\{-2 a_{j i} a_{j k}^{\prime} \phi_{j}\right\},-\left\{a_{j i} \hat{w}_{j}^{\prime}+\hat{w}_{i} a_{i j}^{\prime}\right\}\right)\right] . }
\end{aligned}
$$


On the other hand, the symplectic form is computed as follows. Put $c(\gamma)_{1}:=\gamma^{*}\left(\omega_{1}\right)$ and $c(\gamma)_{2}:=\gamma^{*}\left(\omega_{2}\right)$, where $\omega_{1}$ and $\omega_{2}$ are defined by (3.5) in the proof of Proposition 3.7. We have

$$
\begin{aligned}
c\left(t_{\widehat{\Phi}}(\gamma)\right)_{1}\left(v, v^{\prime}\right)= & {\left[\left(\left\{\operatorname{Tr}\left(\left(\eta_{i j}-a_{i j} A_{i}-a_{i j} \Phi_{i}\right) \circ\left(\eta_{j k}^{\prime}-a_{j k}^{\prime} A_{j}-a_{j k}^{\prime} \Phi_{j}\right)\right)\right\},\right.\right.} \\
& -\left\{\operatorname{Tr}\left(\left(\eta_{i j}-a_{i j} A_{i}-a_{i j} \Phi_{i}\right) \circ\left(v_{j}^{\prime}+\hat{v}_{j}^{\prime}\right)\right)\right. \\
& \left.\left.\left.-\operatorname{Tr}\left(\left(v_{i}+\hat{v}_{i}\right) \circ\left(\eta_{i j}^{\prime}-a_{i j}^{\prime} A_{i}-a_{i j}^{\prime} \Phi_{i}\right)\right)\right\}\right)\right] \\
= & {\left[\left(\left\{-\operatorname{Tr}\left(\left(\eta_{j i}-a_{j i} A_{j}-a_{j i} \Phi_{j}\right) \circ\left(\eta_{j k}^{\prime}-a_{j k}^{\prime} A_{j}-a_{j k}^{\prime} \Phi_{j}\right)\right)\right\},\right.\right.} \\
& -\left\{-\operatorname{Tr}\left(\left(\eta_{j i}-a_{j i} A_{j}-a_{j i} \Phi_{j}\right) \circ\left(v_{j}^{\prime}+\hat{v}_{j}^{\prime}\right)\right)\right. \\
& \left.\left.\left.-\operatorname{Tr}\left(\left(v_{i}+\hat{v}_{i}\right) \circ\left(\eta_{i j}^{\prime}-a_{i j}^{\prime} A_{i}-a_{i j}^{\prime} \Phi_{i}\right)\right)\right\}\right)\right]
\end{aligned}
$$

and

$$
\begin{aligned}
c(\gamma)_{1}\left(v, v^{\prime}\right)= & {\left[\left(\left\{\operatorname{Tr}\left(\left(\eta_{i j}-a_{i j} A_{i}\right) \circ\left(\eta_{j k}^{\prime}-a_{j k}^{\prime} A_{j}\right)\right)\right\},\right.\right.} \\
& \left.\left.-\left\{\operatorname{Tr}\left(\left(\eta_{i j}-a_{i j} A_{i}\right) \circ v_{j}^{\prime}\right)-\operatorname{Tr}\left(v_{i} \circ\left(\eta_{i j}^{\prime}-a_{i j}^{\prime} A_{i}\right)\right)\right\}\right)\right] \\
= & {\left[\left(\left\{-\operatorname{Tr}\left(\left(\eta_{j i}-a_{j i} A_{j}\right) \circ\left(\eta_{j k}^{\prime}-a_{j k}^{\prime} A_{j}\right)\right)\right\},\right.\right.} \\
& \left.\left.-\left\{-\operatorname{Tr}\left(\left(\eta_{j i}-a_{j i} A_{j}\right) \circ v_{j}^{\prime}\right)-\operatorname{Tr}\left(v_{i} \circ\left(\eta_{i j}^{\prime}-a_{i j}^{\prime} A_{i}\right)\right)\right\}\right)\right] .
\end{aligned}
$$

Then we obtain

$$
c\left(t_{\tilde{\Phi}}(\gamma)\right)_{1}\left(v, v^{\prime}\right)-c(\gamma)_{1}\left(v, v^{\prime}\right)=d \widehat{\Phi}_{1}\left(v, v^{\prime}\right) .
$$

Moreover, we also can show that

$$
c\left(t_{\tilde{\Phi}}(\gamma)\right)_{2}\left(v, v^{\prime}\right)-c(\gamma)_{2}\left(v, v^{\prime}\right)=d \widehat{\Phi}_{2}\left(v, v^{\prime}\right) .
$$

Then we obtain that $\left(\Gamma\left(\pi_{\mathfrak{P}_{g, n}(r, e, \boldsymbol{\nu})}\right), c\right)$ is an $\Omega_{\mathfrak{P}_{g, n}(r, e, \boldsymbol{\nu})}^{\geq 1}$-torsor.

\subsection{Extended parabolic connections}

Let $(C, \boldsymbol{t}), \boldsymbol{t}=t_{1}+\cdots+t_{n}$, be an $n$-pointed smooth projective curve of genus $g$ over $\mathbb{C}$ where $t_{1}, \ldots, t_{n}$ are distinct points. Put $D(\boldsymbol{t})=t_{1}+\cdots+t_{n}$. We describe a description of $(\boldsymbol{t}, \boldsymbol{\nu})$ parabolic connection with a quadratic differential in terms of a "integral kernel" on $C \times C$ as in [5] and [6]. Let $p_{1}: C \times C \rightarrow C$ and $p_{2}: C \times C \rightarrow C$ be the first and second projections, respectively. Put $\mathcal{O}_{C}(* D(\boldsymbol{t})):=\lim _{\rightarrow} \mathcal{O}_{C}(m D(\boldsymbol{t}))$, and $\Omega_{C}^{1}(* D(\boldsymbol{t})):=\Omega_{C}^{1} \otimes \mathcal{O}_{C}(* D(\boldsymbol{t}))$. Let $\mathcal{E} \mathrm{nd}^{0}(E) \subset$ $\mathcal{E}$ nd $(E)$ be the subbundle of traceless endmorphisms of $E$. We define sheaves $\mathcal{K}_{D(t)}(E)$ on $C \times C$ as $\mathcal{K}_{D(\boldsymbol{t})}(E):=p_{1}^{*}\left(E \otimes \Omega_{C}^{1}(* D(\boldsymbol{t}))\right) \otimes p_{2}^{*}\left(E^{*} \otimes \Omega_{C}^{1}\right)(2 \Delta)$, where $\Delta \subset C \times C$ is the diagonal. We have a natural injective morphism $\left.\Omega_{C}^{\otimes 2}(* D(t)) \otimes \mathcal{E n d}^{0}(E) \rightarrow \mathcal{K}_{D(\boldsymbol{t})}(E)\right|_{3 \Delta}$. We define a sheaf $\mathcal{E}_{\mathrm{x} C o n n} \operatorname{Con}_{(\boldsymbol{t})}(E)$ on $3 \Delta$ by

$$
\mathcal{E}_{\mathrm{XConn}_{D(\boldsymbol{t})}}(E)=\left\{\left.s \in \mathcal{K}_{D(\boldsymbol{t})}(E)\right|_{3 \Delta} /\left(\Omega_{C}^{\otimes 2}(* D(\boldsymbol{t})) \otimes \mathcal{E}_{\left.\left.\mathrm{nd}^{0}(E)\right)|s|_{\Delta}=\operatorname{Id}_{E}\right\}} .\right.\right.
$$

Note that we can consider $\left.s\right|_{2 \Delta}$ as a connection $\left.s\right|_{2 \Delta}: E \rightarrow E \otimes \Omega_{C}^{1}(* D(\boldsymbol{t}))$. We consider elements of $\mathcal{E x C o n n}_{D(\boldsymbol{t})}(E)$ as pairs of connections and quadratic differentials on $C$ locally. Let $U_{i}$ and $U_{j}$ be open sets of $C$. Let $\left(A_{i}, a_{i}\right)$ be an elements of $\mathcal{E}_{\mathrm{x} C o n n} \operatorname{Con}_{(\boldsymbol{t})}(E)$ on $U_{i}$. Here $A_{i} d f_{i}$ is a connection matrix on $U_{i}$ and $a_{i} d f_{i} \otimes d f_{i} \in H^{0}\left(U_{i}, \Omega_{C}^{\otimes 2}(D(\boldsymbol{t}))\right)$. The transformation of the pair is the following

$$
\begin{aligned}
& \left(A_{i} d f_{i}, a_{i} d f_{i} \otimes d f_{i}\right) \\
& \quad \longmapsto\left(\theta_{i j}^{-1} A_{i} \theta_{i j} d f_{i}+\theta_{i j}^{-1} \frac{d \theta_{i j}}{d f_{i}} d f_{i},\left(a_{i}+\operatorname{Tr}\left(\theta_{i j}^{-1} A_{i} \frac{d \theta_{i j}}{d f_{i}}\right)+\frac{1}{2} \operatorname{Tr}\left(\theta_{i j}^{-1} \frac{d^{2} \theta_{i j}}{d f_{i}^{2}}\right)\right) d f_{i} \otimes d f_{i}\right)
\end{aligned}
$$


on $U_{i} \cap U_{j}$. We may define an $\mathcal{H}_{(C, \boldsymbol{t}, E, \boldsymbol{l})}^{1}$-action on $\mathcal{E}_{\mathrm{xConn}_{D(\boldsymbol{t})}}(E)$ for any parabolic structures $\boldsymbol{l}$ by (4.1).

Let $\nabla: E \rightarrow E \otimes \Omega_{C}^{1}(D(\boldsymbol{t}))$ be a connection. We can define a global section $\nabla_{\mathrm{Ex}}$ of $\mathcal{E}_{\mathrm{xConn}} \operatorname{Cot}_{D(t)}(E)$ associated to $\nabla$ as follows. Take a trivialization of the locally free sheaf $E$ on an open set $U_{i}$ of $C$. Let $A_{i} d f_{i}$ be the connection matrix of $\nabla$ on $U_{i}$. We define $\left.\nabla_{\mathrm{Ex}}\right|_{U_{i}} \in$ $\mathcal{E}_{\mathrm{XConn}}(E)\left(U_{i}\right)$ as

$$
\left(A_{i} d f_{i}, \frac{1}{2} \operatorname{Tr}\left(d\left(A_{i}\right) \otimes d f_{i}\right)+\frac{1}{2} \operatorname{Tr}\left(A_{i} d f_{i} \otimes A_{i} d f_{i}\right)\right)
$$

where $d$ is the exterior derivative. Let $\left(\left(E, \nabla,\left\{l_{j}^{(i)}\right\}\right), \psi\right)$ be a $(\boldsymbol{t}, \boldsymbol{\nu})$-parabolic connection with a quadratic differential. For $(\nabla, \psi)$, we can define a global section $\nabla_{\mathrm{Ex}}+\psi$ of $\mathcal{E}_{\mathrm{x} C o n n} \operatorname{Con}_{D(\boldsymbol{t})}(E)$ by the above construction. We call this description $\left(E, \nabla_{\mathrm{Ex}}+\psi,\left\{l_{j}^{(i)}\right\}\right)$ a $(\boldsymbol{t}, \boldsymbol{\nu})$-extended parabolic connection.

By the identification a parabolic connection with a quadratic differential as an extended parabolic connection, we obtain another $\Omega_{\mathfrak{P}_{g, n}(r, e, \boldsymbol{\nu})}^{1}$-torsor structure on $\Gamma\left(\pi_{\mathfrak{P}_{g, n}(r, e, \boldsymbol{\nu})}\right)$. We take a section $\sigma$ of $\pi_{\mathfrak{P}_{g, n}(r, e, \boldsymbol{\nu})}$ and put $\sigma(p)=\left(\nabla_{p}, \psi_{p}\right)$, where $\nabla_{p}: E \rightarrow E \otimes \Omega_{C}^{1}(D(\boldsymbol{t}))$ is a connection and $\psi_{p} \in H^{0}\left(C, \Omega_{C}^{\otimes 2}(D(\boldsymbol{t}))\right)$ for $p=((C, \boldsymbol{t}),(E, \boldsymbol{l}))$. For a connection $\nabla_{p}: E \rightarrow E \otimes \Omega_{C}^{1}(D(\boldsymbol{t}))$ and $\Phi_{p} \in H^{0}\left(\widetilde{\mathcal{H}}_{p}^{1}\right)$, we define $\psi^{\prime}\left(\nabla_{p}, \Phi_{p}\right) \in H^{0}\left(\mathcal{H}_{p}^{1}\right)$ as follows. We take an affine open covering $\left\{U_{i}\right\}$ of $C$ such that on $U_{i}$ the connection $\left.\nabla_{p}\right|_{U_{i}}$ is described by $d+A_{i} d f_{i}$ and the Higgs field $\left.\Phi_{p}\right|_{U_{i}}$ is described by $\Phi_{i} d f_{i}$. On each $U_{i}$, we define an element $\left.\psi^{\prime}\left(\nabla_{p}, \Phi_{p}\right)\right|_{U_{i}}$ as

$$
\left.\psi^{\prime}\left(\nabla_{p}, \Phi_{p}\right)\right|_{U_{i}}=\left(\Phi_{i} d f_{i}, \operatorname{Tr}\left(\Phi_{i} A_{i} d f_{i} \otimes d f_{i}+\frac{1}{2} \Phi_{i} \Phi_{i} d f_{i} \otimes d f_{i}+\frac{1}{2} d\left(\Phi_{i}\right) \otimes d f_{i}\right)\right) \in \mathcal{F}_{P_{\nu}}^{1}\left(U_{i}\right),
$$

which gives an element $\psi^{\prime}\left(\nabla_{p}, \Phi_{p}\right) \in H^{0}\left(\mathcal{H}_{p}^{1}\right)$. For a 1 -form $\widehat{\Phi}$ on $\mathfrak{P}_{g, n}(r, e, \boldsymbol{\nu})$, we define a translation by

$$
\begin{aligned}
t_{\widehat{\Phi}}^{\prime}: & \Gamma\left(\pi_{\mathfrak{P}_{g, n}(r, e, \boldsymbol{\nu})}\right) \longrightarrow \Gamma\left(\pi_{\mathfrak{P}_{g, n}(r, e, \boldsymbol{\nu})}\right), \\
& \sigma(p)=\left(\nabla_{p}, \psi_{p}\right) \longmapsto t_{\widehat{\Phi}}^{\prime}(\sigma)(p):=\left(\nabla_{p}+\kappa\left(\widehat{\Phi}_{p}\right), \psi_{p}-\left(\widehat{\Phi}_{p}-\psi^{\prime}\left(\nabla_{p}, \kappa\left(\widehat{\Phi}_{p}\right)\right)\right)\right) .
\end{aligned}
$$

By the translation, we have another $\Omega_{\mathfrak{P}_{g, n}(r, e, \boldsymbol{\nu})}^{1}$-torsor structure on $\Gamma\left(\pi_{\mathfrak{P}_{g, n}(r, e, \boldsymbol{\nu})}\right)$.

\section{Acknowledgements}

The author is supported by Grant-in-Aid for JSPS Research Fellows Number 18J00245. He is grateful to the anonymous referees' suggestions which helped to improve the paper.

\section{References}

[1] Atiyah M.F., Bott R., The Yang-Mills equations over Riemann surfaces, Philos. Trans. Roy. Soc. London Ser. A 308 (1983), 523-615.

[2] Beilinson A., Bernstein J., A proof of Jantzen conjectures, in I.M. Gel'fand Seminar, Adv. Soviet Math., Vol. 16, Amer. Math. Soc., Providence, RI, 1993, 1-50.

[3] Beilinson A., Kazhdan D., Flat projective connection, unpublished.

[4] Beilinson A.A., Schechtman V.V., Determinant bundles and Virasoro algebras, Comm. Math. Phys. 118 (1988), 651-701.

[5] Ben-Zvi D., Biswas I., Theta functions and Szegő kernels, Int. Math. Res. Not. 2003 (2003), 1305-1340, math.AG/0211441.

[6] Ben-Zvi D., Biswas I., Opers and theta functions, Adv. Math. 181 (2004), 368-395, math.AG/0204301. 
[7] Ben-Zvi D., Frenkel E., Geometric realization of the Segal-Sugawara construction, in Topology, Geometry and Quantum Field Theory, London Math. Soc. Lecture Note Ser., Vol. 308, Cambridge University Press, Cambridge, 2004, 46-97, math.AG/0301206.

[8] Bloch S., Esnault H., Relative algebraic differential characters, math.AG/9912015.

[9] Boalch P., Symplectic manifolds and isomonodromic deformations, Adv. Math. 163 (2001), 137-205.

[10] Faltings G., Stable G-bundles and projective connections, J. Algebraic Geom. 2 (1993), 507-568.

[11] Goldman W.M., The symplectic nature of fundamental groups of surfaces, Adv. Math. 54 (1984), $200-225$.

[12] Hitchin N., The self-duality equations on a Riemann surface, Proc. London Math. Soc. 55 (1987), 59-126.

[13] Hitchin N., Geometrical aspects of Schlesinger's equation, J. Geom. Phys. 23 (1997), 287-300.

[14] Hurtubise J., On the geometry of isomonodromic deformations, J. Geom. Phys. 58 (2008), 1394-1406, arXiv:0804.0249.

[15] Inaba M.-A., Moduli of parabolic connections on curves and the Riemann-Hilbert correspondence, J. Algebraic Geom. 22 (2013), 407-480, math.AG/0602004.

[16] Inaba M.-A., Iwasaki K., Saito M.-H., Moduli of stable parabolic connections, Riemann-Hilbert correspondence and geometry of Painlevé equation of type VI, Part I, Publ. Res. Inst. Math. Sci. 42 (2006), 987-1089, math.AG/0309342.

[17] Iwasaki K., Moduli and deformation for Fuchsian projective connections on a Riemann surface, J. Fac. Sci. Univ. Tokyo Sect. IA Math. 38 (1991), 431-531.

[18] Komyo A., Hamiltonian structures of isomonodromic deformations on moduli spaces of parabolic connections, arXiv:1611.03601.

[19] Krichever I., Isomonodromy equations on algebraic curves, canonical transformations and Whitham equations, Mosc. Math. J. 2 (2002), 717-752, hep-th/0112096.

[20] Narasimhan M.S., Seshadri C.S., Stable and unitary vector bundles on a compact Riemann surface, Ann. of Math. 82 (1965), 540-567.

[21] Simpson C.T., Moduli of representations of the fundamental group of a smooth projective variety. I, Inst. Hautes Études Sci. Publ. Math. (1994), 47-129.

[22] Simpson C.T., Moduli of representations of the fundamental group of a smooth projective variety. II, Inst. Hautes Études Sci. Publ. Math. (1994), 5-79. 